\title{
Complex Dynamics in Predator-prey Models with Nonmonotonic Functional Response and Harvesting
}

\author{
J. Huang ${ }^{1 *}$, J. Chen ${ }^{2}$, Y. Gong ${ }^{1}$, W. Zhang ${ }^{3}$ \\ ${ }^{1}$ School of Mathematics and Statistics, Central China Normal University \\ Wuhan, Hubei 430079, P. R. China \\ ${ }^{2}$ Department of Mathematics, University of Miami, Coral Gables, FL 33124-4250, USA \\ 3 School of Mathematics and Statistics, Northeast Normal University \\ Changchun, Jilin 130024, P. R. China
}

\begin{abstract}
In this paper we study the complex dynamics of predator-prey systems with nonmonotonic functional response and harvesting. When the harvesting is constant-yield for prey, it is shown that various kinds of bifurcations, such as saddle-node bifurcation, degenerate Hopf bifurcation, and Bogdanov-Takens bifurcation, occur in the model as parameters vary. The existence of two limit cycles and a homoclinic loop is established by numerical simulations. When the harvesting is seasonal for both species, sufficient conditions for the existence of an asymptotically stable periodic solution and bifurcation of a stable periodic orbit into a stable invariant torus of the model are given. Numerical simulations are carried out to demonstrate the existence of bifurcation of a stable periodic orbit into an invariant torus and transition from invariant tori to periodic solutions, respectively, as the amplitude of seasonal harvesting increases.
\end{abstract}

Keywords and phrases: predator-prey model, constant-yield harvesting, seasonal harvesting, Bogdanov-Takens bifurcation, degenerate Hopf bifurcation, periodic solution, invariant torus

Mathematics Subject Classification: 34C23, 34C25, 34A26

\section{Introduction}

Predator-prey models have been refined so as to better reflect the complex interaction of the different populations. A central role in the nature and stability of predator-prey population dynamics is the functional response of predators to the prey, which describes the change in the density of the prey attacked per unit time per predator as the prey density changes. In general the functional response depends on many factors, for example, prey density, the efficiency with which predators can search out and kill the prey, the handling time, induced defenses in prey (Hammill et al. [10]), etc. Various types of functional response functions, such as Lotka-Volterra function, Holling type II function, and Holling type III function, have been frequently used in the literature. A nonmonotone function

$$
p(x)=\frac{m x}{a x^{2}+b x+1},
$$

\footnotetext{
${ }^{*}$ Corresponding author. E-mail: hjc@mail.ccnu.edu.cn
} 
where $m$ and $a$ are positive constants and $b>-2 \sqrt{a}$, is called the Monod-Haldane (Andrews [1], Ruan and Xiao [14]) or the generalized Holling type IV (Taylor [16], Collings [6]) functional response and can be used to describe the phenomenon of "inhibition" in microbial dynamics and "group defence" in population dynamics. When $b=0$, the function is called the Holling type IV functional response in the literature (Ruan and Xiao [14]). Predator-prey systems with nonmonotone functional response have been extensively studied by many authors (see Ruan and Xiao [14], Wolkowicz [17], and Zhu, Campbell and Wolkowicz [19] and references cited therein).

In the last three decades, how to harvest the interacting species properly has attracted great attention, many scientists have begun to investigate the effect of harvesting on the dynamics of predator-prey systems and the role of harvesting in the management of renewable resources. One question arises naturally: Can appropriate harvest on the interacting species stabilize the system such that both the prey and predators coexist or increase the survival of predators when the prey exists "group defence" phenomenon? May et al. [12] have proposed two types of harvesting regimes to qualitatively describe the effect of harvest: constant-effort harvesting, described by a constant multiplication of the size of the population under harvest, and constant-yield harvesting, described by a constant independent of the size of the population under harvest. Nevertheless, in some cases, harvesting is often effected by seasonal change, so it is more reasonable to consider seasonal harvesting, which was considered in single species models in Hirsch et al. [9] and Brauer and Sánchez [5]. The existence and the number of periodic orbits by calculating the Poincaré map are discussed in [9]. Brauer and Sánchez [5] have shown that the behavior of the model with periodic harvesting is similar to that of the model with no harvesting, but with an asymptotically stable periodic solution instead of an asymptotically stable equilibrium. Nevertheless, we have known little about periodic harvesting of interacting populations, which is an interesting topic to be explored ([5]).

The purpose of this paper is to study the following system with nonmonotonic functional response and seasonal harvesting

$$
\begin{aligned}
& \dot{x}=r x\left(1-\frac{x}{K}\right)-\frac{m x y}{c+x^{2}}-\left(H+\gamma_{1}^{\prime} \sin (2 \pi t)\right), \\
& \dot{y}=y\left(\frac{\beta x}{c+x^{2}}-\delta\right)-\gamma_{2}^{\prime}(1+\sin (2 \pi t)),
\end{aligned}
$$

where $x$ and $y$ are functions of time representing population densities of the prey and predators, respectively, $\frac{m x}{c+x^{2}}$ is the Holling type-IV functional response which is nonmonotonic in the first quadrant, $K, \delta, r, c, \beta, m$ and $H$ are all positive constants and stand for the carrying capacity of the prey, the death rate of the predator, the intrinsic maximum growth rate of the prey, the half-saturation constant, the conversion rate of prey to predator, the capturing rate and the constant-yield prey harvesting, respectively. $H+\gamma_{1}^{\prime} \sin (2 \pi t)$ and $\gamma_{2}^{\prime}(1+\sin (2 \pi t))$ describe the seasonal harvesting effort on the prey and predators population, respectively, in which $\gamma_{1}^{\prime} \leq H, \gamma_{2}^{\prime} \geq 0$ are the amplitudes of seasonal prey and predator harvesting, respectively. Ruan and Xiao [14] studied system (1.1) with $H=0$ and $\gamma_{1}^{\prime}=\gamma_{2}^{\prime}=0$, and showed that it undergoes various kinds of bifurcations, including saddle-node bifurcation, supercritical and subcritical Hopf bifurcation and Bogdanov-Takens bifurcation.

For mathematical simplicity, we first nondimensionalize system (1.1) with the following scaling

$$
\tau \rightarrow r t, X \rightarrow \frac{x}{K}, Y \rightarrow \frac{m y}{r K^{2}},
$$

and still denote $\tau, X, Y$ by $t, x, y$, respectively. System (1.1) takes the form

$$
\begin{aligned}
& \dot{x}=x(1-x)-\frac{x y}{a+x^{2}}-\left(h+\gamma_{1} \sin \left(\frac{2 \pi}{r} t\right)\right), \\
& \dot{y}=y\left(\frac{\mu x}{a+x^{2}}-d\right)-\gamma_{2}\left(1+\sin \left(\frac{2 \pi}{r} t\right)\right),
\end{aligned}
$$

where $a=\frac{c}{K^{2}}, \mu=\frac{\beta}{r K}, h=\frac{H}{K r}, \gamma_{1}=\frac{\gamma_{1}^{\prime}}{K r}, \gamma_{2}=\frac{m \gamma_{2}^{\prime}}{r^{2} K^{2}}, d=\frac{\delta}{r}$ and $\gamma_{1} \leq h$. For simplicity, in the following we keep the biological implications of parameters $a, \mu, h, \gamma_{1}, \gamma_{2}$ and $d$ the same as $c, \beta, H, \gamma_{1}^{\prime}, \gamma_{2}^{\prime}$ and $\delta$, respectively.

We first consider system (1.2) with only constant-yield prey harvesting (that is $\gamma_{1}=\gamma_{2}=0$ ) and show that several kinds of bifurcation phenomena, including saddle-node bifurcation, degenerate Hopf 
bifurcation, and Bogdanov-Takens bifurcation (i.e. cusp bifurcation of codimension 2), occur as the model parameters vary. The existence of two limit cycles, a homoclinic loop, different bifurcation curves and phase portraits of the model are also given, which reveal far richer dynamics compared to the case with no harvesting. Then we consider system (1.2) with seasonal harvesting in both species and give sufficient conditions for the existence of an asymptotically stable periodic solution and bifurcation of a stable periodic orbit into a stable invariant torus of the model. Numerical simulations of model (1.2), including bifurcation diagram, phase portraits, and the attractor of the Poincaré map, demonstrate that the model undergoes bifurcation of a stable periodic solution into an invariant torus and transition from invariant tori to periodic solutions as the amplitude of seasonal harvesting increases.

The paper is organized as follows. In section 2, we study the existence of various types of equilibria in model (1.2) with only constant-yield harvesting. We also describe the phase portraits and the biological ramifications of our results. In section 3, we discuss possible bifurcations of the model (1.2) with only constant-yield prey harvesting depending on all parameters and show that the model exhibits saddle-node bifurcation, degenerate Hopf bifurcation, and Bogdanov-Takens bifurcation in terms of the original model parameters. In section 4, the existence of an asymptotically stable periodic solution and bifurcation of a stable periodic orbit into an invariant torus in system (1.2) are established by theoretical analysis and numerical simulations. The paper ends with a brief discussion in section 5 .

\section{Stability with constant-yield harvesting}

In this section we perform a qualitative analysis of model (1.2) with constant-yield prey harvesting, that is $\gamma_{1}=\gamma_{2}=0$. We rewrite it as

$$
\begin{aligned}
& \dot{x}=x(1-x)-\frac{x y}{a+x^{2}}-h \triangleq f_{1}(x, y), \\
& \dot{y}=y\left(\frac{\mu x}{a+x^{2}}-d\right) \triangleq f_{2}(x, y),
\end{aligned}
$$

where $a, h, d$ and $\mu$ are positive parameters. From the viewpoint of biology, we are interested in the dynamics of system (2.1) in the closed first quadrant $R_{+}^{2}$. Thus, we only consider the biologically meaningful initial conditions

$$
x(0) \geq 0, y(0) \geq 0 .
$$

It is obvious that the solution of (2.1) with nonnegative initial conditions exists and is unique. It is also easy to see that the $x$-axis is invariant under the flow. Nevertheless, this is not the case on the $y$-axis. All solutions touching the $y$-axis cross out of the first quadrant. Thus, the first quadrant is no longer positively invariant under the flow generated by system (2.1), which makes the qualitative analysis of system (2.1) more challenging.

\subsection{Equilibria}

System (2.1) has the following possible equilibria:

$$
A=\left(x_{1}, 0\right), B=\left(x_{2}, 0\right), C=\left(x_{3}, y_{3}\right), D=\left(x_{4}, y_{4}\right), E=\left(\frac{1}{2}, 0\right), F=(\sqrt{a}, 2 a-2 a \sqrt{a}-2 h \sqrt{a}),
$$

where

$$
\begin{gathered}
x_{1}=\frac{1-\sqrt{1-4 h}}{2}, x_{2}=\frac{1+\sqrt{1-4 h}}{2}, \\
x_{3}=\frac{\mu-\sqrt{\mu^{2}-4 a d^{2}}}{2 d}, x_{4}=\frac{\mu+\sqrt{\mu^{2}-4 a d^{2}}}{2 d}, \\
y_{3}=\frac{\left(a+x_{3}^{2}\right)\left(h_{1}-h\right)}{x_{3}}, y_{4}=\frac{\left(a+x_{4}^{2}\right)\left(h_{2}-h\right)}{x_{4}}, \\
h_{1}=x_{3}-x_{3}^{2}, h_{2}=x_{4}-x_{4}^{2} .
\end{gathered}
$$


TABLE 1. Equilibria of the system (2.1)

\begin{tabular}{|l|c|}
\hline Cases & Equilibria \\
\hline$h>\frac{1}{4}$ & none \\
$h=\frac{1}{4}$ & $E$ \\
$0<h<\frac{1}{4}$ and $\mu^{2}-4 a d^{2}<0$ & $A$ and $B$ \\
$\sqrt{a}-a \leq h<\frac{1}{4}$ and $\mu^{2}-4 a d^{2}=0$ & $A$ and $B$ \\
$\max \left\{h_{1}, h_{2}\right\}<h<\frac{1}{4}$ and $\mu^{2}-4 a d^{2}>0$ & $A$ and $B$ \\
$0<h<\sqrt{a}-a$ and $\mu^{2}-4 a d^{2}=0$ & $A, B$ and $F$ \\
$h_{2}<h<h_{1}$ and $\mu^{2}-4 a d^{2}>0$ & $A, B$ and $C$ \\
$h_{1}<h<h_{2}$ and $\mu^{2}-4 a d^{2}>0$ & $A, B$ and $D$ \\
$0<h<\min \left\{h_{1}, h_{2}\right\}$ and $\mu^{2}-4 a d^{2}>0$ & $A, B, C$ and $D$ \\
\hline
\end{tabular}

Theorem 2.1. The equilibria of the system (2.1), according to the values of parameters, are given in Table 1.

Proof. An equilibrium of the system (2.1) has coordinates $\left(x_{0}, y_{0}\right)$, where $x_{0}, y_{0}$ are solutions of the system with unknown $(x, y)$

$$
\begin{aligned}
& x(1-x)-\frac{x y}{a+x^{2}}-h=0, \\
& y\left(\frac{\mu x}{a+x^{2}}-d\right)=0,
\end{aligned}
$$

such that $x_{0} \geq 0, y_{0} \geq 0$, by the second equation of (2.2), one has $y=0$ or $d x^{2}-\mu x+a d=0$.

(1) For $y=0$, the first equation of (2.2) gives:

$$
x^{2}-x+h=0,
$$

whose discriminant is $\Delta_{1}:=1-4 h$. It follows that: (i) when $h>\frac{1}{4}$, there is no equilibrium on $x$-axis; (ii) when $h=\frac{1}{4}$, then $\left(\frac{1}{2}, 0\right)$ is a double equilibrium; (iii) when $0<h<\frac{1}{4}$, then (2.3) has two positive real roots $x_{1}$ and $x_{2}$, which correspond to equilibria $\left(x_{1}, 0\right)$ and $\left(x_{2}, 0\right)$ of system $(2.1)$.

(2) For:

$$
d x^{2}-\mu x+a d=0,
$$

whose discriminant is $\Delta_{2}:=\mu^{2}-4 a d^{2}$. It follows that: (i) when $\mu^{2}-4 a d^{2}<0,(2.4)$ does not have any real roots; (ii) when $\mu^{2}-4 a d^{2}=0$, then the only real root of (2.4) is $x_{0}=\frac{\mu}{2 d}=\sqrt{a}$, which corresponds to solution $F=(\sqrt{a}, 2 a-2 a \sqrt{a}-2 h \sqrt{a})$ of equations (2.2). From the expression of $y_{3}$, we can see that $y_{3}<0$ if $h>\sqrt{a}-a ; y_{3}=0$ if $h=\sqrt{a}-a$ and $F$ equals one of $A$ and $B ; y_{3}>0$ if $h<\sqrt{a}-a$ and $F$ is in the interior of $R_{+}^{2}$; (iii) when $\mu^{2}-4 a d^{2}>0$, then (2.4) has two positive real roots $x_{3}$ and $x_{4}$, which correspond to solutions $C=\left(x_{3}, y_{3}\right), D=\left(x_{4}, y_{4}\right)$ of equations (2.2). From the expressions of $y_{3}$ and $y_{4}$, we can see that $C=\left(x_{3}, y_{3}\right)$ is not in $R_{+}^{2}$ if $h_{1}<h<h_{2}$ and $\mu^{2}-4 a d^{2}>0$ because $y_{3}<0 ; D=\left(x_{4}, y_{4}\right)$ is not in $R_{+}^{2}$ if $h_{2}<h<h_{1}$ and $\mu^{2}-4 a d^{2}>0$ because $y_{4}<0$.

From Theorem 2.1, we can see that when $h>\frac{1}{4}$, system (2.1) has no equilibria, and $\frac{d x}{d t}<0$ in $R_{+}^{2}$. The dynamics of system (2.1) are trivial in $R_{+}^{2}$ and all orbits in $R_{+}^{2}$ will cross the $y$-axis and go out of $R_{+}^{2}$ in finite time (see also Fig. 1(a)). This implies that the prey species goes extinct, which in turn causes the extinction of predators. Biological over-harvesting occurs. When $0<h \leq \frac{1}{4}$, system (2.1) has some equilibria, and there exist some initial values such that the population of the prey in system (2.1) persists. Thus, $h_{M S Y}=\frac{1}{4}$ for system (2.1) from Theorem 2.1. Hence, the constant-yield harvesting rate $h$ must satisfy $0<h<\frac{1}{4}$. Mathematically, we will see that the complex dynamics of system (2.1) occur when $0<h<\frac{1}{4}$. 


\subsection{Stability}

In this section we consider the dynamics of system (2.1) in the neighborhood of each equilibrium. The linearization of system (2.1) at these equilibria is determined by the Jacobian matrix

$$
D f(x, y)=\left(\begin{array}{cc}
1-2 x-\frac{y\left(a-x^{2}\right)}{\left(a+x^{2}\right)^{2}} & \frac{-x}{a+x^{2}} \\
\frac{\mu y\left(a-x^{2}\right)}{\left(a+x^{2}\right)^{2}} & -d+\frac{\mu x}{a+x^{2}}
\end{array}\right)
$$

where $f=\left(f_{1}, f_{2}\right)^{T}, x$ and $y$ are coordinates of these equilibria, respectively.

In the following theorem, we only consider equilibria $A, B, C$ and $D$ and will consider $E, F$ later.

Theorem 2.2. The types of equilibria $A, B, C$ and $D$ are, according to the values of parameters, given in Table 2. The phase portraits of system (2.1) without any equilibria or with two boundary equilibria are shown in Figure 1.

TABle 2. Types of the equilibria of the system (2.1)

\begin{tabular}{|c|c|c|}
\hline Cases & equilibria & Type \\
\hline $\max \{0, \sqrt{a}-a\}<h<\frac{1}{4}$ and $\mu^{2}-4 a d^{2} \leq 0$ & $A$ and $B$ & $A$ is a hyperbolic saddle, \\
\hline$h=\sqrt{a}-a, a<\frac{1}{4}$ and $\mu^{2}-4 a d^{2}=0$ & $A$ and $B$ & $\begin{array}{l}B \text { is a nyperbolic stable node } \\
A \text { is a nonhyperbolic saddle, } \\
B \text { is a hyperbolic stable node }\end{array}$ \\
\hline$h=\sqrt{a}-a, \frac{1}{4}<a<1$ and $\mu^{2}-4 a d^{2}=0$ & $A$ and $B$ & $\begin{array}{l}A \text { is a hyperbolic saddle, } \\
B \text { is a nonhyperbolic stable node }\end{array}$ \\
\hline $\begin{array}{l}\max \left\{h_{1}, h_{2}\right\}<h<\frac{1}{4}, \mu^{2}-4 a d^{2}>0 \\
\text { and } x_{3}<\frac{1}{2}<x_{4}\end{array}$ & $A$ and $B$ & $\begin{array}{l}A \text { is a hyperbolic unstable node, } \\
B \text { is a hyperbolic saddle }\end{array}$ \\
\hline $\begin{array}{l}\max \left\{h_{1}, h_{2}\right\}<h<\frac{1}{4}, \mu^{2}-4 a d^{2}>0 \\
\text { and } x_{3}>\frac{1}{2}\left(\text { or } x_{4}<\frac{1}{2}\right)\end{array}$ & $A$ and $B$ & $\begin{array}{l}A \text { is a hyperbolic saddle } \\
B \text { is a hyperbolic stable node }\end{array}$ \\
\hline$h_{2}<h<h_{1}$ and $\mu^{2}-4 a d^{2}>0$ & $A, B$ and $C$ & $\begin{array}{l}A \text { and } B \text { are hyperbolic saddles, } \\
C \text { is an anti-saddle }\end{array}$ \\
\hline$h_{1}<h<h_{2}$ and $\mu^{2}-4 a d^{2}>0$ & $A, B$ and $D$ & $\begin{array}{l}A \text { is a hyperbolic unstable node, } \\
B \text { is a hyperbolic stable node, } \\
D \text { is a hyperbolic saddle }\end{array}$ \\
\hline $0<h<\min \left\{h_{1}, h_{2}\right\}$ and $\mu^{2}-4 a d^{2}>0$ & $A, B, C$ and $D$ & $\begin{array}{l}A \text { and } D \text { are hyperbolic saddles, } \\
B \text { is a hyperbolic stable node, } \\
C \text { is an anti-saddle }\end{array}$ \\
\hline
\end{tabular}

Proof. (1) We firstly consider the types of equilibria $A=\left(x_{1}, 0\right)$ and $B=\left(x_{2}, 0\right)$, where $x_{1}=$ $\frac{1-\sqrt{1-4 h}}{2}, x_{2}=\frac{1+\sqrt{1-4 h}}{2}, 0<h<\frac{1}{4}$. By $(2.5)$, we have

$$
D f(A)=\left(\begin{array}{cc}
1-2 x_{1} & \frac{-x_{1}}{a+x_{1}^{2}} \\
0 & -d+\frac{\mu x_{1}}{a+x_{1}^{2}}
\end{array}\right), D f(B)=\left(\begin{array}{cc}
1-2 x_{2} & \frac{-x_{1}}{a+x_{2}^{2}} \\
0 & -d+\frac{\mu x_{2}}{a+x_{2}^{2}}
\end{array}\right) .
$$

It is clear that $1-2 x_{i}$ and $-d+\frac{\mu x_{i}}{a+x_{i}^{2}}(i=1,2)$ are the eigenvalues of $D f(A)$ and $D f(B)$. Moreover, we can see that $1-2 x_{1}=\sqrt{1-4 h}>0,1-2 x_{2}=-\sqrt{1-4 h}<0$, and it is not difficult to check the following results:

Case 1. $-d+\frac{\mu x_{i}}{a+x_{i}^{2}}<0$ if $\mu^{2}-4 a d^{2}<0$

Case 2. $-d+\frac{\mu x_{i}^{2}}{a+x_{i}^{2}}<0$ and $x_{i} \neq \frac{\mu}{2 d}$ if $\mu^{2}-4 a d^{2}=0$ and $\max \{0, \sqrt{a}-a\}<h<\frac{1}{4}$;

Case 3. $-d+\frac{\mu x_{1}^{2}}{a+x_{1}^{2}}=0,-d+\frac{\mu x_{2}}{a+x_{2}^{2}}<0$ if $\mu^{2}-4 a d^{2}=0, h=\sqrt{a}-a$ and $0<a<\frac{1}{4}$; 
Case 4. $-d+\frac{\mu x_{1}}{a+x_{1}^{2}}<0,-d+\frac{\mu x_{2}}{a+x_{2}^{2}}=0$ if $\mu^{2}-4 a d^{2}=0, h=\sqrt{a}-a$ and $\frac{1}{4}<a<1$;

Case 5. $-d+\frac{\mu x_{i}}{a+x_{i}^{2}}>0$ if $\mu^{2}-4 a d^{2}>0, \max \left\{h_{1}, h_{2}\right\}<h<\frac{1}{4}$ and $x_{3}<\frac{1}{2}<x_{4}$;

Case 6. $-d+\frac{\mu x_{i}{ }^{2}}{a+x_{i}^{2}}<0$ if $\mu^{2}-4 a d^{2}>0, \max \left\{h_{1}, h_{2}\right\}<h<\frac{1}{4}$ and $x_{3}>\frac{1}{2}\left(\right.$ or $x_{4}<\frac{1}{2}$ );

Case 7. $-d+\frac{\mu x_{1}^{2}}{a+x_{1}^{2}}<0$ and $-d+\frac{\mu x_{2}}{a+x_{2}^{2}}>0$ if $\mu^{2}-4 a d^{2}>0$ and $h_{2}<h<h_{1}$;

Case 8. $-d+\frac{\mu x_{1}}{a+x_{1}^{2}}>0$ and $-d+\frac{\mu x_{2}}{a+x_{2}^{2}}<0$ if $\mu^{2}-4 a d^{2}>0$ and $h_{1}<h<h_{2}$;

Case $9 .-d+\frac{\mu x_{i}}{a+x_{i}^{2}}<0$ if $\mu^{2}-4 a d^{2}>0$ and $0<h<\min \left\{h_{1}, h_{2}\right\}$.

The types of the singular points $A$ and $B$ are obvious except the case 3 and case 4 in which the singular points $A=(\sqrt{a}, 0)\left(a<\frac{1}{4}\right)$ and $B=(\sqrt{a}, 0)\left(\frac{1}{4}<a<1\right)$ are nonhyperbolic. Now we investigate the types of $A$ and $B$ when they are nonhyperbolic.

Firstly, we translate $(\sqrt{a}, 0)$ to the origin and expand system $(2.1)$ in a power series around the origin. Let $X=x-\sqrt{a}, Y=y$. Then system (2.1) can be rewritten as

$$
\begin{aligned}
& \dot{X}=(1-2 \sqrt{a}) X-\frac{1}{2 \sqrt{a}} Y-X^{2}+\frac{1}{4 a \sqrt{a}} X^{2} Y+P_{1}(X, Y), \\
& \dot{Y}=-\frac{\mu}{4 a \sqrt{a}} X^{2} Y+Q_{1}(X, Y),
\end{aligned}
$$

where $P_{1}(X, Y)$ and $Q_{1}(X, Y)$ are $C^{\infty}$ functions at least of order four in $(X, Y)$.

Next, making the affine transformation

$$
X=-\frac{1}{2 \sqrt{a}} x+y, Y=(2 \sqrt{a}-1) x,
$$

we can rewrite system (2.6) as follows

$$
\begin{aligned}
& \dot{x}=-\frac{\mu}{16 a^{2} \sqrt{a}} x^{3}+P_{2}(x, y), \\
& \dot{y}=(1-2 \sqrt{a}) y-\frac{1}{4 a} x^{2}+\frac{1}{\sqrt{a}} x y-y^{2}+Q_{2}(x, y),
\end{aligned}
$$

where $P_{2}(x, y)$ and $Q_{2}(x, y)$ are $C^{\infty}$ functions at least of order four and order three in $(x, y)$, respectively.

Let $\tau=(1-2 \sqrt{a}) t$, then system $(2.7)$ can be rewritten as

$$
\begin{aligned}
& \dot{x}=-\frac{\mu}{16 a^{2} \sqrt{a}-32 a^{3}} x^{3}+P_{3}(x, y), \\
& \dot{y}=y-\frac{1}{4 a-8 a \sqrt{a}} x^{2}+\frac{1}{\sqrt{a}-2 a} x y-\frac{1}{1-2 \sqrt{a}} y^{2}+Q_{3}(x, y),
\end{aligned}
$$

where $P_{3}(x, y)$ and $Q_{3}(x, y)$ are $C^{\infty}$ functions at least of order four and order three in $(x, y)$, respectively. Consequently, the coefficient of $x^{3}$ in the first equation of $(2.8)$ is $-\frac{\mu}{16 a^{2} \sqrt{a}-32 a^{3}} \neq 0\left(<0\right.$ if $0<a<\frac{1}{4}$; $>0$ if $\left.\frac{1}{4}<a<1\right)$, so by Theorem 7.1 in Zhang et al. [20], the equilibrium $A=(\sqrt{a}, 0)$ is a nonhyperbolic saddle if $0<a<\frac{1}{4} ; B=(\sqrt{a}, 0)$ is a nonhyperbolic stable node if $\frac{1}{4}<a<1$.

(2) We then consider the types of the singular points $C=\left(x_{3}, y_{3}\right)$ and $D=\left(x_{4}, y_{4}\right)$. By (2.5), the determinants of $D f(C)$ and $D f(D)$ are

$$
\operatorname{Det}(\operatorname{Df}(C))=\left(\frac{\mu}{x_{3}}-2 d\right)\left(x_{3}-x_{3}^{2}-h\right), \operatorname{Det}(\operatorname{Df}(D))=\left(\frac{\mu}{x_{4}}-2 d\right)\left(x_{4}-x_{4}^{2}-h\right),
$$

respectively. Since $x_{3}<\frac{\mu}{2 d}<x_{4}$ and $x_{i}-x_{i}^{2}-h=\frac{x_{i} y_{i}}{a+x_{i}^{2}}>0(i=3,4)$, we have $\operatorname{Det}(\operatorname{Df}(C))>0$ and $\operatorname{Det}(D f(D))<0$. Hence, $D$ is a hyperbolic saddle and $C$ is an anti-saddle.

Remark 2.3. Since we have let $\tau=(1-2 \sqrt{a}) t$, then $t: 0 \mapsto-\infty$ as $\tau: 0 \mapsto+\infty$ if $\frac{1}{4}<a<1$. Hence, $B(\sqrt{a}, 0)\left(\frac{1}{4}<a<1\right)$ is a stable node as $t: 0 \mapsto+\infty$ when it is an unstable node as $\tau: 0 \mapsto+\infty$.

Remark 2.4. From Fig. 1(b), we can see that there exists one separatrix which converges to the boundary equilibrium $A$, the prey species will tend to extinction as the initial density lies to the left of the separatrix, and the predator species will tend to extinction as the initial density lies to the right of the 
(a)

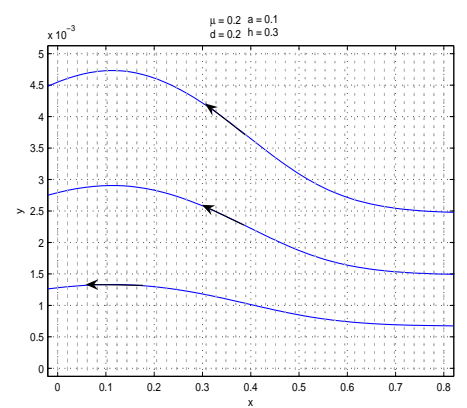

(b)

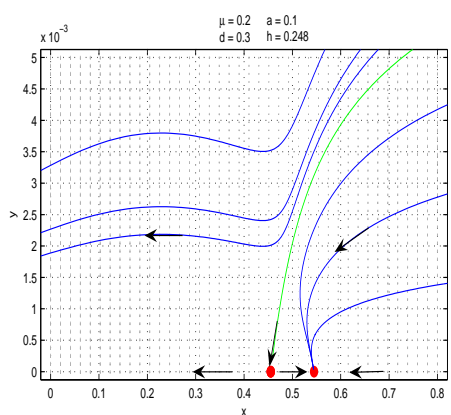

(c)

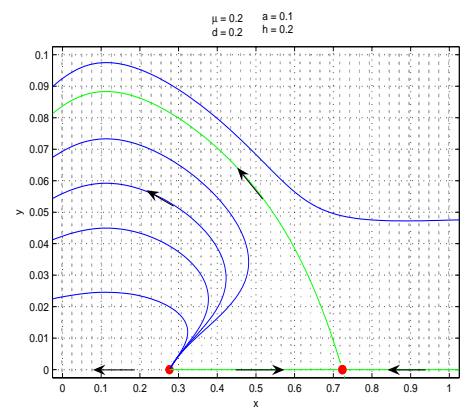

FIGURE 1. The phase portraits of system (2.1) without any equilibria or with two boundary equilibria. (a) No equilibria; (b)(c) Two boundary equilibria.

separatrix. Therefore, sufficient enrichment of the environment leads to the extinction of predators for all initial values to the right of the separatrix, which strongly supports the so-called paradox of enrichment. From Fig. 1(c), the prey will tend to extinction for almost all initial values. Hence, system (2.1) is not persistent when it has only two boundary equilibria.

Theorem 2.5. when $h=\frac{1}{4}$, system (2.1) has a unique singular point $E=\left(\frac{1}{2}, 0\right)$, and

(i) $E$ is a saddle-node if $\frac{2 \mu}{4 a+1} \neq d$, it is attracting (repelling) if $d>\frac{2 \mu}{4 a+1}\left(d<\frac{2 \mu}{4 a+1}\right)$;

(ii) $E$ is a nonhyperbolic saddle if $\frac{2 \mu}{4 a+1}=d$ and $a>\frac{1}{4}$;

(iii) $E$ is a saddle-node if $\frac{2 \mu}{4 a+1}=d$ and $a=\frac{1}{4}$ (that is $\mu=d$ );

(iv) $E$ is a degenerate singular point and $S_{\delta}\left(\left(\frac{1}{2}, 0\right)\right)$ consists of one hyperbolic sector and one elliptic sector if $\frac{2 \mu}{4 a+1}=d$ and $a<\frac{1}{4}$, where $S_{\delta}\left(\left(\frac{1}{2}, 0\right)\right)$ is the neighborhood of $\left(\frac{1}{2}, 0\right)$ with sufficient small radius $\delta$. The phase portraits are shown in Figure 2.

Proof. By (2.5), we have

$$
D f(E)=\left(\begin{array}{lc}
0 & \frac{-2}{4 a+1} \\
0 & -d+\frac{2 \mu}{4 a+1}
\end{array}\right) .
$$

It follows that the eigenvalues of $D f(E)$ are $\lambda_{1}=0$ and $\lambda_{2}=\frac{2 \mu}{4 a+1}-d$.

The translation $(X, Y)=\left(x-\frac{1}{2}, y\right)$ brings the singularity $\left(\frac{1}{2}, 0\right)$ to the origin. In a neighborhood of the origin, system (2.1) becomes

$$
\begin{aligned}
& \dot{X}=\frac{-2}{4 a+1} Y-X^{2}-\frac{16 a-4}{(4 a+1)^{2}} X Y-\frac{8-384 a^{2}-64 a}{(4 a+1)^{4}} X^{2} Y+P_{1}(X, Y), \\
& \dot{Y}=\left(\frac{2 \mu}{4 a+1}-d\right) Y-\frac{16 \mu a-4 \mu}{(4 a+1)^{2}} X Y+\frac{8 \mu-384 a^{2} \mu-64 a \mu}{(4 a+1)^{4}} X^{2} Y+Q_{1}(X, Y),
\end{aligned}
$$

where $P_{1}(X, Y)$ and $Q_{1}(X, Y)$ are $C^{\infty}$ functions at least of order four in $(X, Y)$.

(1) If $\lambda_{2} \neq 0$, let $X=x-\frac{2}{4 a+1} y, Y=\left(\frac{2 \mu}{4 a+1}-d\right) y$, then system (2.9) becomes

$$
\begin{aligned}
& \dot{x}=-x^{2}+\left(2 \alpha_{1}-\alpha_{2} \beta_{1}+\alpha_{1} \beta_{2}\right) x y+\left(\alpha_{1} \alpha_{2}-\alpha_{1}^{2}-\frac{\alpha_{1}^{2} \beta_{2}}{\beta_{1}}\right) y^{2}+P_{2}(x, y), \\
& \dot{y}=\beta_{1} y+\beta_{2} x y+\alpha_{1} \beta_{2} y^{2}+Q_{2}(x, y),
\end{aligned}
$$

where $P_{2}(x, y)$ and $Q_{2}(x, y)$ are $C^{\infty}$ functions at least of order three in $(x, y)$, and

$$
\alpha_{1}=\frac{-2}{4 a+1}, \alpha_{2}=\frac{16 a-4}{(4 a+1)^{2}}, \beta_{1}=\left(\frac{2 \mu}{4 a+1}-d\right), \beta_{2}=\frac{16 \mu a-4 \mu}{(4 a+1)^{2}}
$$


(a)

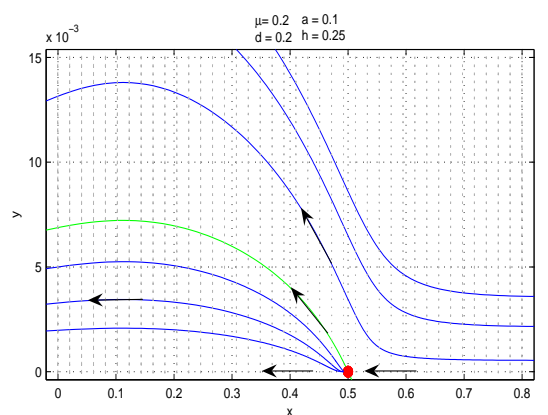

(c)

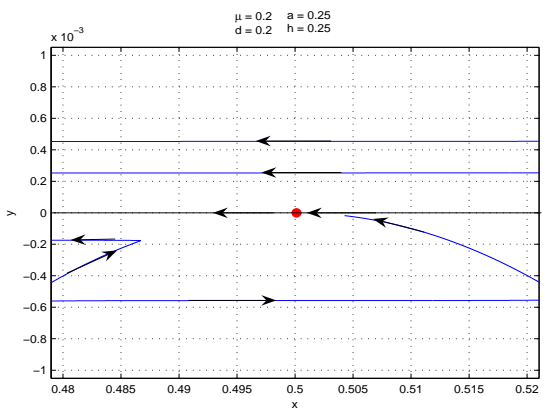

(b)

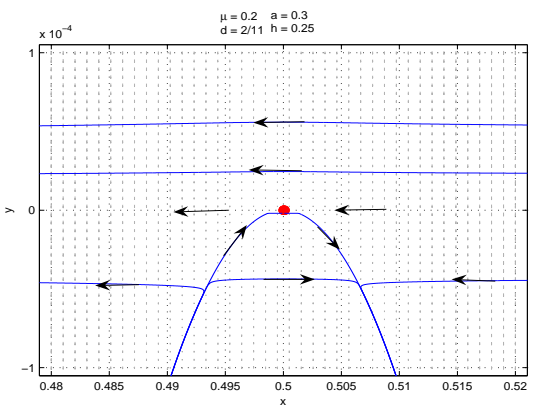

(d)

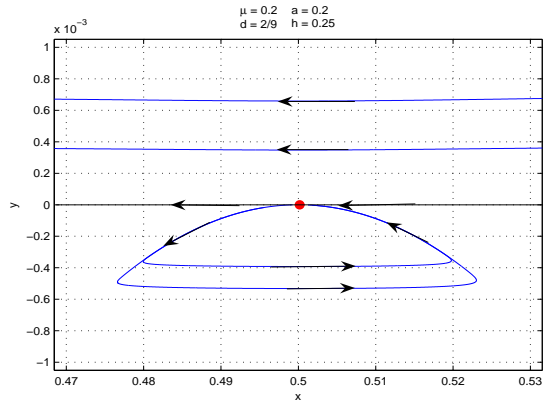

Figure 2. The phase portraits of system (2.1) with only one boundary equilibrium when $h=\frac{1}{4}$. (a) $\frac{2 \mu}{4 a+1} \neq d$; (b) $\frac{2 \mu}{4 a+1}=d$ and $a>\frac{1}{4}$; (c) $\frac{2 \mu}{4 a+1}=d$ and $a=\frac{1}{4}$; (d) $\frac{2 \mu}{4 a+1}=d$ and $a<\frac{1}{4}$.

Next, introducing a new time variable $\tau$ by $\tau=\beta_{1} t$ and still denoting $\tau$ with $t$, we obtain

$$
\begin{aligned}
& \dot{x}=-\frac{1}{\beta_{1}} x^{2}+\frac{\left(2 \alpha_{1}-\alpha_{2} \beta_{1}+\alpha_{1} \beta_{2}\right)}{\beta_{1}} x y+\frac{\left(\alpha_{1} \alpha_{2}-\alpha_{1}^{2}-\frac{\alpha_{1}^{2} \beta_{2}}{\beta_{1}}\right)}{\beta_{1}} y^{2}+P_{3}(x, y), \\
& \dot{y}=y+\frac{\beta_{2}}{\beta_{1}} x y+\frac{\alpha_{1} \beta_{2}}{\beta_{1}} y^{2}+Q_{3}(x, y) .
\end{aligned}
$$

Since the coefficient of $x^{2}$ in the first equation of system (2.11) is $\frac{-1}{\beta_{1}}=\frac{-1}{\lambda_{2}} \neq 0$, by Theorem 7.1 in Zhang et al. [20], we know that the equilibrium $E=\left(\frac{1}{2}, 0\right)$ is a saddle-node, which is attracting (repelling) if $d>\frac{2 \mu}{4 a+1}\left(d<\frac{2 \mu}{4 a+1}\right)$.

(2) If $\lambda_{2}=0$, let $\tau=\frac{-2}{4 a+1} t$, then (2.9) becomes

$$
\begin{aligned}
& \dot{X}=Y+P_{4}(X, Y), \\
& \dot{Y}=Q_{4}(X, Y),
\end{aligned}
$$

where $P_{4}(X, Y)$ and $Q_{4}(X, Y)$ are $C^{\infty}$ functions at least of order two in $(X, Y)$.

Let $Y+P_{4}(X, Y)=0$, we can obtain the implicit function

$$
Y=\phi(X)=-\frac{4 a+1}{2} X^{2}+(4 a-1) X^{3}+I_{4}(X),
$$

where $I_{4}(X)$ is a $C^{\infty}$ function at least of order four. Replacing $Y$ with $\phi(X)$, we have

$$
\psi(X) \triangleq Q_{4}(X, \phi(X))=\mu(4 a-1) X^{3}-8 a \mu X^{4}+I_{5}(X),
$$




$$
\delta(X) \triangleq \frac{\partial P_{4}}{\partial X}(X, \phi(X))+\frac{\partial Q_{4}}{\partial Y}(X, \phi(X))=\left(4 a+1-\frac{8 \mu a-2 \mu}{4 a+1}\right) X+I_{2}(X),
$$

where $I_{5}(X)$ and $I_{2}(X)$ are $C^{\infty}$ functions at least of order five and order two, respectively.

Denote

$$
a_{3}=\mu(4 a-1), a_{4}=-8 a \mu, b_{1}=4 a+1-\frac{8 \mu a-2 \mu}{4 a+1} .
$$

From Theorems 7.2, 7.3 and their deduction in Zhang et al. [20], the type of $\left(\frac{1}{2}, 0\right)$ is as follows: if $a>\frac{1}{4}$, then $a_{3}>0,\left(\frac{1}{2}, 0\right)$ is a degenerate saddle; if $a=\frac{1}{4}$, then $a_{3}=0, a_{4}<0$ and $b_{1} \neq 0,\left(\frac{1}{2}, 0\right)$ is a saddle-node; if $a<\frac{1}{4}$, then $a_{3}<0, b_{1}>0$ and $b_{1}^{2}+8 a_{3} \geq 0, S_{\delta}\left(\left(\frac{1}{2}, 0\right)\right)$ consists of one hyperbolic sector and one elliptic sector.

Remark 2.6. In Theorem $2.5, \dot{x}=-\left(x-\frac{1}{2}\right)^{2}-\frac{x y}{a+x^{2}}<0$ in $R_{+}^{2}$, which implies that the prey species may go extinction as time increases for almost all initial values, which is shown in Fig. 2. Hence, for the persistence of the ecosystem, the equilibrium of the greatest interest would be an equilibrium interior to the first quadrant.

Theorem 2.7. If $0<h<\sqrt{a}-a$ and $\mu^{2}-4 a d^{2}=0$, then system (2.1) has three equilibria, which are $A, B$ and $F$, and no closed orbits in $R_{+}^{2}$. Moreover,

(i) If $a \neq \frac{1}{4}, A, B$ and $F$ are a hyperbolic saddle, a hyperbolic stable node and a saddle-node, respectively, and $F$ is attracting (repelling) if $a>\frac{1}{4}\left(a<\frac{1}{4}\right)$;

(ii) If $a=\frac{1}{4}, A, B$ and $F$ are a hyperbolic saddle, a hyperbolic stable node and a cusp of codimension 2, respectively. The phase portraits of system (2.1) are given in Figure 3.

(a)

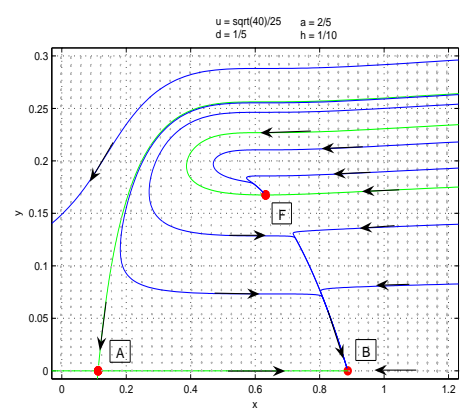

(b)

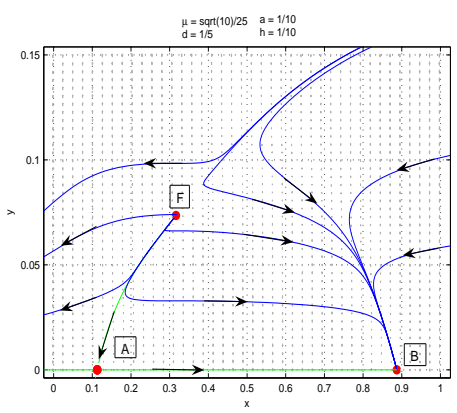

(c)

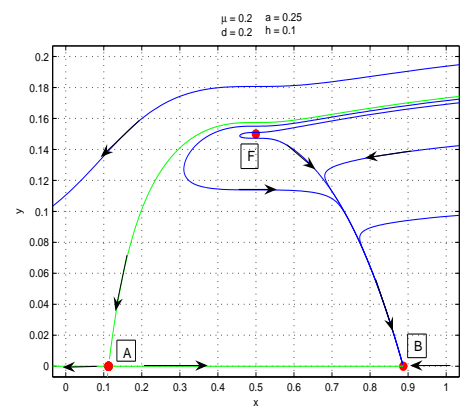

Figure 3 . The phase portraits of system (2.1) when $0<h<\sqrt{a}-a$ and $\mu^{2}-4 a d^{2}=0$. (a) $a>\frac{1}{4}$; (b) $0<a<\frac{1}{4}$; (c) $a=\frac{1}{4}$.

Proof. (1) The types of $A$ and $B$ can be obtained from Theorem 2.2 directly.

(2) If $a=\frac{1}{4}, F$ is a cusp of codimension 2, we will give the proof in section 3.3.

(3) If $a \neq \frac{1}{4}, F$ is a saddle-node. The translation $(X, Y)=(x-\sqrt{a}, y-2 a+2 a \sqrt{a}+2 h \sqrt{a})$ brings the singularity $(\sqrt{a}, 2 a-2 a \sqrt{a}-2 h \sqrt{a})$ to the origin. In a neighborhood of the origin, system $(2.1)$ becomes

$$
\begin{aligned}
& \dot{X}=a_{10} X+a_{01} Y+a_{20} X^{2}+P_{1}(X, Y), \\
& \dot{Y}=b_{20} X^{2}+Q_{1}(X, Y)
\end{aligned}
$$


where $P_{1}(X, Y)$ and $Q_{1}(X, Y)$ are $C^{\infty}$ functions at least of order three in $(X, Y)$, and

$$
a_{10}=1-2 \sqrt{a}, a_{01}=\frac{-1}{2 \sqrt{a}}, a_{20}=\frac{\sqrt{a}-3 a-h}{2 a}, b_{20}=\frac{-\mu(\sqrt{a}-a-h)}{2 a} .
$$

Let $X=a_{01} x+y, Y=-a_{10} x$, then $(2.13)$ becomes

$$
\begin{aligned}
& \dot{x}=-\frac{b_{20} a_{01}^{2}}{a_{10}} x^{2}-\frac{2 b_{20} a_{01}}{a_{10}} x y-\frac{b_{20}}{a_{10}} y^{2}+P_{2}(x, y), \\
& \dot{y}=a_{10} y+a_{20} a_{01}^{2} x^{2}+2 a_{20} a_{01} x y+a_{20} y^{2}+Q_{2}(x, y),
\end{aligned}
$$

where $P_{2}(x, y)$ and $Q_{2}(x, y)$ are $C^{\infty}$ functions at least of order three in $(x, y)$.

By introducing a new time variable $\tau$ by $\tau=a_{10} t$ and still denoting $\tau$ with $t$, we obtain

$$
\begin{aligned}
& \dot{x}=-\frac{b_{20} a_{01}^{2}}{a_{10}^{2}} x^{2}-\frac{2 b_{20} a_{01}}{a_{10}^{2}} x y-\frac{b_{20}}{a_{10}^{2}} y^{2}+P_{3}(x, y), \\
& \dot{y}=y+\frac{a_{20} a_{01}^{2}}{a_{10}} x^{2}+\frac{2 a_{20} a_{01}}{a_{10}} x y+\frac{a_{20}}{a_{10}} y^{2}+Q_{3}(x, y),
\end{aligned}
$$

where $P_{3}(x, y)$ and $Q_{3}(x, y)$ are $C^{\infty}$ functions at least of order three in $(x, y)$. The coefficient of $x^{2}$ in the first equation of system (2.15) is $-\frac{b_{20} a_{01}^{2}}{a_{10}^{2}} \neq 0$ since $h<\sqrt{a}-a$, then the equilibrium $F=$ $(\sqrt{a}, 2 a-2 a \sqrt{a}-2 h \sqrt{a})$ is a saddle-node, which is attracting (repelling) if $a>\frac{1}{4}\left(a<\frac{1}{4}\right)$. Since a closed orbit must include some equilibria in its interior and the total sum of indices of these equilibria should equal one, system (2.1) does not have a closed orbit in $R_{+}^{2}$ under the conditions of Theorem 2.7.

Theorem 2.8. If $\mu^{2}-4 a d^{2}>0$ and $0<h<h_{1}$, then $C=\left(x_{3}, y_{3}\right)$ is a positive equilibrium of system (2.1). Let $h_{3}(a, d, \mu)=\frac{a x_{3}^{2}-2 x_{3}^{3}+3 x_{3}^{4}}{a-x_{3}^{2}}=\frac{-\left(\mu-\sqrt{\mu^{2}-4 a d^{2}}\right)^{2}\left(4 a d^{2}+(3 \mu-2 d)\left(-\mu+\sqrt{\mu^{2}-4 a d^{2}}\right)\right)}{4 d^{2}\left(4 a d^{2}+\mu\left(-\mu+\sqrt{\mu^{2}-4 a d^{2}}\right)\right)}$, then

(i) $\left(x_{3}, y_{3}\right)$ is a hyperbolic stable focus (or node) if $h<h_{3}$;

(ii) $\left(x_{3}, y_{3}\right)$ is a weak focus or center if $h=h_{3}$;

(iii) $\left(x_{3}, y_{3}\right)$ is a hyperbolic unstable focus (or node) if $h>h_{3}$.

Proof. From Theorem 2.2 we can see that $\operatorname{Det}(\operatorname{Df}(C))>0$. On the other hand,

$$
\operatorname{Tr}(D f(C))=1-2 x_{3}-\frac{y_{3}\left(a-x_{3}^{2}\right)}{\left(a+x_{3}^{2}\right)^{2}}=\frac{a-x_{3}^{2}}{x_{3}\left(a+x_{3}^{2}\right)}\left(h-h_{3}\right)=\frac{1}{d}\left(\frac{\mu}{x_{3}}-2 d\right)\left(h-h_{3}\right),
$$

since $0<x_{3}<\frac{\mu}{2 d}$, hence $\operatorname{Tr}(D f(C))<0$ if $0<h<\min \left\{h_{3}, h_{1}\right\} ; \operatorname{Tr}(D f(C))=0$ if $0<h=h_{3}<h_{1}$; $\operatorname{Tr}(D f(C))>0$ if $0<h_{3}<h<h_{1}$.

\section{Bifurcations with constant-yield harvesting}

In this section, we investigate the bifurcations of system (2.1) with constant-yield harvesting.

\subsection{Saddle-node bifurcations}

From Theorems 2.1, 2.2 and 2.5 we know that

$$
S N_{1}=\left\{(a, d, \mu, h): h=\frac{1}{4}, \frac{2 \mu}{4 a+1} \neq d, a, d, \mu>0\right\}
$$

is a saddle-node bifurcation surface. When the parameters pass from one side of the surface to the other side, the number of equilibria of system (2.1) changes from zero to two, and the two equilibria which are boundary equilibria are hyperbolic saddle and node. This is the first saddle-node bifurcation surface of the model. The biological interpretation for the first saddle-node bifurcation is that $h_{M S Y}=\frac{1}{4}$, the prey species is driven to extinction, and the system collapses for $h>\frac{1}{4}$, but the prey species does not go to extinction for some initial data when $0<h<\frac{1}{4}$. 
From Theorem 2.1, 2.2 and 2.7, on the other hand, we know that the surface

$$
S N_{2}=\left\{(a, d, \mu, h): \mu^{2}-4 a d=0,0<h<\sqrt{a}-a, a \neq \frac{1}{4}, a, d, \mu>0\right\}
$$

is also a saddle-node bifurcation surface, which is the second saddle-node bifurcation the model undergoes. The saddle-node bifurcation yields two positive equilibria. This implies that there exists a critical constant-yield harvesting $h_{0}=\sqrt{a}-a$ such that the predator species goes either extinct or out of $R_{+}^{2}$ in finite time when the constant-yield harvesting $h$ is greater than $h_{0}$, and coexistence for model (2.1) is certain in the form of a positive equilibrium for certain choices of initial values when $\mu^{2}-4 a d=0$ and $0<h<h_{0}$.

\subsection{Degenerate Hopf bifurcation}

In this section, we transform system (2.1) to a generalized Lienard system to study the order of the Hopf bifurcation, because the calculation of the Lyapunov coefficients of such a system can be easier. The following lemma is Theorem 5.1 in Lamontagne et al. [11], similar results or methods can also be found in Etoua and Rousseau [8] and Xiao and Zhu [18].

Lemma 3.1. For a generalized Lienard system

$$
\begin{aligned}
& \dot{x}=-y, \\
& \dot{y}=g(x)+y f(x),
\end{aligned}
$$

where

$$
g(x)=\sum_{i=1}^{+\infty} A_{i} x^{i}, f(x)=\sum_{j=1}^{+\infty} B_{j} x^{j}
$$

When $A_{1}>0$, then the formulas of the first two Lyapunov coefficients take the useful form

$$
L_{1}=\frac{B_{2} A_{1}-A_{2} B_{1}}{8 A_{1}^{\frac{3}{2}}}, L_{2}=\frac{1}{16 A_{1}^{\frac{5}{2}}}\left(\frac{5}{3} A_{2} A_{3} B_{1}-\frac{5}{3} A_{1} A_{2} B_{3}+B_{4} A_{1}^{2}-A_{1} A_{4} B_{1}\right) .
$$

From Theorem 2.8, we know that the positive equilibrium $\left(x_{3}, y_{3}\right)$ of system (2.1) is a center-type nonhyperbolic equilibrium when $\mu^{2}-4 a d^{2}>0$ and $0<h=h_{3}<h_{1}$. Hence, system (2.1) may undergo Hopf bifurcation. In this section, we will give a full analysis of the Hopf bifurcation which is summarized in the following theorem. Firstly, we deduce some conditions from the necessary conditions for the Hopf bifurcation, which will be used in the following theorem and proof:

$$
\begin{gathered}
h=h_{3}=\frac{a x_{3}^{2}-2 x_{3}^{3}+3 x_{3}^{4}}{a-x_{3}^{2}}<h_{1}=x_{3}-x_{3}^{2} \Longrightarrow x_{3}<\frac{1}{2}, \\
0<h=h_{3} \Longrightarrow 3 x_{3}^{2}-2 x_{3}+a>0, \\
\mu^{2}-4 a d^{2}>0 \Longrightarrow x_{3}=\frac{\mu-\sqrt{\mu^{2}-4 a d^{2}}}{2 d}<\sqrt{a} .
\end{gathered}
$$

Theorem 3.2. When $\mu^{2}-4 a d^{2}>0,0<h=h_{3}<h_{1}$, then

(i) the equilibrium $\left(x_{3}, y_{3}\right)$ is a stable weak focus of multiplicity 1 if $L_{11}<0$;

(ii) the equilibrium $\left(x_{3}, y_{3}\right)$ is an unstable weak focus of multiplicity 1 if $L_{11}>0$;

(ii) the equilibrium $\left(x_{3}, y_{3}\right)$ is a stable weak focus of multiplicity 2 if $L_{11}=0$, where

$$
L_{11}=a^{3}-9 a^{2} x_{3}^{2}-3 x_{3}^{6}+a x_{3}^{3}\left(4+3 x_{3}\right) .
$$


Proof. We firstly rescale the time by dividing (2.1) by $\frac{x}{a+x^{2}}$. Since $\frac{x}{a+x^{2}}>0$ for all $x>0$, neither the orientation of trajectories nor the number of periodic solutions will change. Next we bring $\left(x_{3}, y_{3}\right)$ to origin by the translation $(X, Y)=\left(x-x_{3}, y-y_{3}\right)$, then system $(2.1)$ has the form

$$
\begin{aligned}
& \dot{X}=P(X)-Y, \\
& \dot{Y}=\left(Y+y_{3}\right)\left(\mu-\frac{a d+d\left(X+x_{3}\right)^{2}}{X+x_{3}}\right),
\end{aligned}
$$

where

$$
P(X)=-\left(X+x_{3}\right)^{3}+\left(X+x_{3}\right)^{2}-(a+h)\left(X+x_{3}\right)+a-y_{3}-\frac{a h}{X+x_{3}} .
$$

The generalized Lienard system is simply obtained by letting $(x, y)=(X, Y-P(X))$

$$
\begin{aligned}
& \dot{x}=-y, \\
& \dot{y}=g(x)+y f(x),
\end{aligned}
$$

where

$$
g(x)=\left(P(x)+y_{3}\right)\left(\mu-\frac{a d+d\left(x+x_{3}\right)^{2}}{x+x_{3}}\right), f(x)=P^{\prime}(x)+\mu-\frac{a d+d\left(x+x_{3}\right)^{2}}{x+x_{3}},
$$

and $P^{\prime}(x)$ is the derivative of $P(x)$ with respect to $x$.

Following Lemma 3.1 and eliminating $h, d$ by $h=h_{3}=\frac{a x_{3}^{2}-2 x_{3}^{3}+3 x_{3}^{4}}{a-x_{3}^{2}}$ and $d=\frac{\mu x_{3}}{a+x_{3}^{2}}$, respectively, we obtain

$$
\begin{gathered}
A_{1}=\frac{\mu}{x_{3}}\left(1-2 x_{3}\right)\left(a+x_{3}^{2}\right), A_{2}=\frac{a \mu\left(-1+2 x_{3}\right)\left(a+x_{3}^{2}\right)}{x_{3}^{2}\left(a-x_{3}^{2}\right)}, \\
A_{3}=\frac{\mu}{x_{3}^{3}\left(a^{2}-x_{3}^{4}\right)}\left(a^{3}\left(1-3 x_{3}\right)+a^{2}\left(5-9 x_{3}\right) x_{3}^{2}+x_{3}^{6}-3 x_{3}^{7}+a x_{3}^{4}\left(-3+7 x_{3}\right)\right), \\
A_{4}=\frac{-\mu}{x_{3}^{4}\left(a^{2}-x_{3}^{4}\right)}\left(a^{3}\left(1-4 x_{3}\right)+a^{2}\left(7-11 x_{3}\right) x_{3}^{2}+x_{3}^{7}+2 a\left(-1+x_{3}\right) x_{3}^{4}\right), \\
A_{5}=\frac{a \mu}{x_{3}^{5}\left(a^{2}-x_{3}^{4}\right)}\left(a^{2}\left(1-5 x_{3}\right)+a\left(9-14 x_{3}\right) x_{3}^{2}+\left(-2+3 x_{3}\right) x_{3}^{4}\right), \\
B_{1}=\frac{1}{-a^{2} x_{3}+x_{3}^{5}}\left(2 a^{3}+a^{2}\left(-6 x_{3}+14 x_{3}^{2}-\mu\right)+2 a x_{3}^{2}\left(-2 x_{3}+3 x_{3}^{2}+\mu\right)-x_{3}^{4}\left(-2 x_{3}+6 x_{3}^{2}+\mu\right)\right), \\
B_{2}=\frac{1}{-a^{2} x_{3}^{2}+x_{3}^{6}}\left(-3 a^{3}-3 x_{3}^{6}+a^{2}\left(6 x_{3}-9 x_{3}^{2}+\mu\right)-a x_{3}^{2}\left(-6 x_{3}+9 x_{3}^{2}+\mu\right)\right), \\
B_{3}=\frac{a}{-a^{2} x_{3}^{3}+x_{3}^{7}}\left(4 a^{2}+a\left(-8 x_{3}+16 x_{3}^{2}-\mu\right)+x_{3}^{2}\left(-8 x_{3}+12 x_{3}^{2}+\mu\right)\right), \\
B_{4}=\frac{a}{-a^{2} x_{3}^{4}+x_{3}^{8}}\left(-5 a^{2}+a\left(10 x_{3}-20 x_{3}^{2}+\mu\right)-x_{3}^{2}\left(-10 x_{3}+15 x_{3}^{2}+\mu\right)\right) .
\end{gathered}
$$

By the formulas of the Lyapunov coefficients in Lemma 3.1, we have

$$
L_{1}=\frac{a^{3}-9 a^{2} x_{3}^{2}-3 x_{3}^{6}+a x_{3}^{3}\left(4+3 x_{3}\right)}{8\left(-a x_{3}+x_{3}^{3}\right)^{2} \sqrt{\frac{\mu\left(1-2 x_{3}\right)\left(a+x_{3}^{2}\right)}{x_{3}}}},
$$

from conditions (3.2) and (3.4), we can see that $L_{1}$ is well defined, and the sign of $L_{1}$ is the same as:

$$
L_{11}=a^{3}-9 a^{2} x_{3}^{2}-3 x_{3}^{6}+a x_{3}^{3}\left(4+3 x_{3}\right) .
$$

Therefore, when $\mu^{2}-4 a d^{2}>0,0<h=h_{3}<h_{1}$ and $L_{11}<0,\left(x_{3}, y_{3}\right)$ is a stable weak focus of multiplicity 1 ; when $\mu^{2}-4 a d^{2}>0,0<h=h_{3}<h_{1}$ and $L_{11}>0,\left(x_{3}, y_{3}\right)$ is an unstable weak focus of multiplicity 1 ; when $\mu^{2}-4 a d^{2}>0,0<h=h_{3}<h_{1}$ and $L_{11}=0,\left(x_{3}, y_{3}\right)$ is a weak focus of multiplicity at least 2 . 
Next, we prove that the order of the weak focus $\left(x_{3}, y_{3}\right)$ is 2 if $L_{11}=0$ and $h=h_{3}$. Once again, by the formulas of the Lyapunov coefficients in Lemma 3.1, and using the condition $L_{11}=0$, we have

$$
L_{2}=\frac{-\mu x_{3}^{2}\left(-1+2 x_{3}\right)\left(a+x_{3}^{2}\right)}{6\left(-a^{2}+x_{3}^{4}\right)^{3}\left(\frac{-\mu\left(-1+2 x_{3}\right)\left(a+x_{3}^{2}\right)}{x_{3}}\right)^{\frac{3}{2}}} L_{22},
$$

where

$$
\begin{aligned}
L_{22}= & 12 x_{3}^{5}\left(-4+25 x_{3}+68 x_{3}^{2}\right)-a x_{3}^{2}\left(-64+355 x_{3}+1346 x_{3}^{2}+720 x_{3}^{3}\right) \\
& +a^{2}\left(4-193 x_{3}+742 x_{3}^{2}+2352 x_{3}^{3}\right) .
\end{aligned}
$$

It is clear that the sign of $L_{2}$ is determined by $L_{22}$ when $L_{11}=0$ and $h=h_{3}$. From conditions (3.2) and (3.4) it is easy to see that $L_{2}<0$ if $L_{22}>0$, next we prove $L_{22}$ is strictly positive for $\mu^{2}-4 a d^{2}>0$, $0<h=h_{3}<h_{1}$ and $L_{11}=0$.

Firstly, under the hypothesis $L_{11}=0$ it is sufficient to take the resultant of the numerators of $L_{11}$ and $L_{22}$ with respect to $a$

$$
\text { resultant }\left[\operatorname{num}\left(L_{11}\right), \operatorname{num}\left(L_{22}\right), a\right]=36 x_{3}^{13}\left(2 x_{3}-1\right)^{2}\left(1+4 x_{3}\right)^{3}\left(16-45 x_{3}+12 x_{3}^{2}+348 x_{3}^{3}\right),
$$

by condition (3.2) it is not difficult to prove that the above resultant can not equal zero when $0<x_{3}<\frac{1}{2}$, that is to say that $L_{11}$ and $L_{22}$ never vanish simultaneously under the conditions of the theorem.

Secondly, it remains to show that $L_{22}$ is strictly positive. Since $L_{11}$ and $L_{22}$ are not zero simultaneously, $L_{22}$ does not change sign when $L_{11}=0$ (by the intermediate value theorem). Let $a=\frac{343}{400}, x_{3}=\frac{7}{20}$, then $L_{11}=0$, and $L_{22}=\frac{170473401}{2500000} \doteq 68.1894>0$. Hence, $L_{2}$ is negative when $L_{1}=0$ and $h=h_{3}$.

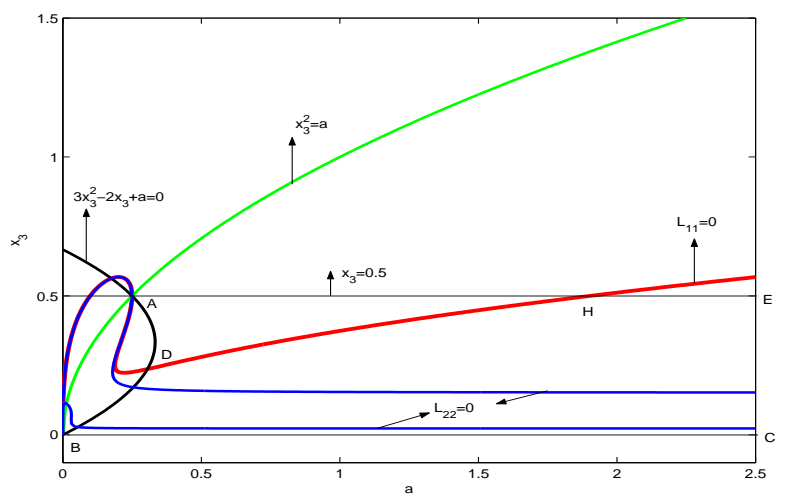

FiguRE 4. Illustrating the proof of Theorem 3.2.

Remark 3.3. From (3.2)-(3.4), in order to illustrate the admissible region for $L_{11}$ and $L_{22}$, we plot the curves $x_{3}=\frac{1}{2}, x_{3}=\sqrt{a}, 3 x_{3}^{2}-2 x_{3}+a=0, L_{11}=0$ and $L_{22}=0$ in $\left(a, x_{3}\right)$-plane in Fig. 4, from which we can see that the admissible region for $L_{11}$ and $L_{22}$ is under the line $A E\left(x_{3}=\frac{1}{2}\right)$, on the right of parabolic curve $\widehat{A B}\left(3 x_{3}^{2}-2 x_{3}+a=0\right)$ and above the line $B C\left(x_{3}=0\right)$, we denote this region by $\widehat{A D C E} A$, it is clear that $L_{11}=0$ and $L_{22}=0$ have no common roots in this region, in fact $L_{22}>0$ if $L_{11}=0$ and $\left(a, x_{3}\right)$ is located in this region. One also can check that $L_{11}<0$ when $\left(a, x_{3}\right)$ is located in the interior of region $\widehat{A D H A}$, and $L_{11}>0$ when $\left(a, x_{3}\right)$ is located in the interior of region $\widehat{D B C E H D}$. Hence, $\left(x_{3}, y_{3}\right)$ is a stable weak focus of multiplicity 1 if $\left(a, x_{3}\right)$ is located in the interior of region $\widehat{A D H A}$ and $h=h_{3}$; $\left(x_{3}, y_{3}\right)$ is an unstable weak focus of multiplicity 1 if $\left(a, x_{3}\right)$ is located in the interior of region $\widehat{D B C E H D}$ and $h=h_{3} ;\left(x_{3}, y_{3}\right)$ is a stable weak focus of multiplicity 2 if $\left(a, x_{3}\right)$ on the curve $\widehat{D H}$ and $h=h_{3}$. 
By the theory of Hopf bifurcation in [20] and [13], we obtain the following results.

Theorem 3.4. Suppose that $\mu^{2}-4 a d^{2}>0,0<h=h_{3}<h_{1}$ hold.

(i) If $L_{11}<0$, then system (2.1) has a stable limit cycle arising from the supercritical Hopf bifurcation as $h$ increases from $h_{3}$;

(ii) If $L_{11}>0$, then system (2.1) has an unstable limit cycle arising from the subcritical Hopf bifurcation as $h$ decreases from $h_{3}$;

(iii) If $L_{11}=0$, then system (2.1) has two limit cycles arising from the degenerate Hopf bifurcation as $h$ decreases from $h_{3}$ and $L_{11}$ increases from 0 , and the inner is unstable and the outer is stable.

(a)

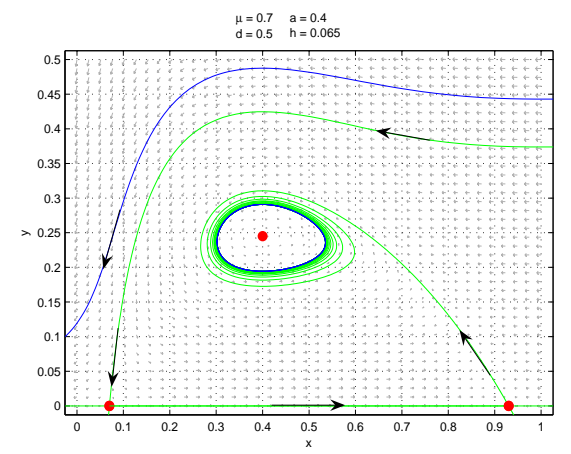

(b)

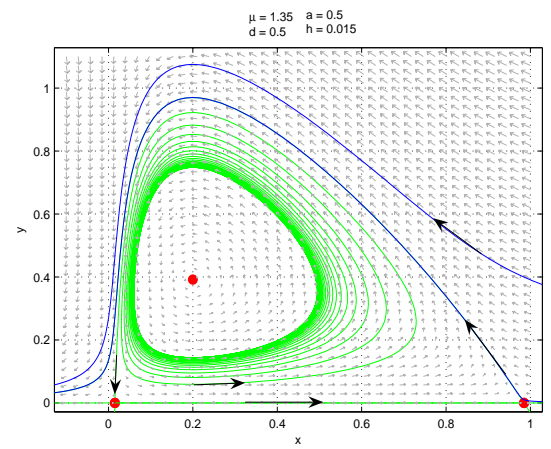

Figure 5. (a) A stable limit cycle created by the supercritical Hopf bifurcation; (b) An unstable limit cycle created by the subcritical Hopf bifurcation.

In Fig. 5, by incorporating the above analysis and using numerical simulations we present the supercritical and subcritical Hopf bifurcations of codimension 1. In Fig. 5(a), we fix $a=0.4, \mu=$ $0.7, d=0.5, h=0.065$, then $L_{1}=-1.39605<0$ and $h=0.065>h_{3}=\frac{4}{75} \doteq 0.0533$, and there exists a stable limit cycle in a small neighborhood of the equilibria $(0.4,0.245)$. In Fig. 5(b), we fix $a=0.5, \mu=1.35, d=0.5, h=0.015$, then $L_{1}=0.531358>0$ and $h=0.015<h_{3}=\frac{11}{575} \doteq 0.019$, and an unstable limit cycle appears in a small neighborhood of the equilibria $(0.2,0.3915)$.

In Fig. 6, we show the degenerate Hopf bifurcation and the existence of two limit cycles, where the unstable one is in the interior of the stable one. We firstly choose $x_{3}=\frac{7}{20}$, and get $a_{0}=\frac{343}{400}$ by $L_{11}\left(a_{0}, x_{3}\right)=0$, that is $\left(a_{0}, x_{3}\right) \in \widehat{D H}$, then we perturb $a$ such that $a=\frac{343}{400}+\frac{7}{400}$, we can get $h_{3}\left(a, x_{3}\right)=\frac{1519}{17200}, L_{1}=\frac{1812}{103544}>0$ and $L_{22}=\frac{5722161543}{80000000}>0\left(\right.$ i.e. $\left.L_{2}<0\right)$, secondly, we perturb $h$ such that $h=\frac{1519}{17200}-\frac{11}{1436200}$, we can get $y_{3} \doteq 0.396702, \operatorname{tr}\left(D f\left(x_{3}, y_{3}\right)\right) \doteq-0.0000165$ and $\operatorname{det}\left(\operatorname{Df}\left(x_{3}, y_{3}\right)\right) \doteq$ 0.171009 , in the last, we let $d=\frac{1}{5}$ and get $\mu=\frac{57}{100}$. There exist two limit cycles in the small neighborhood of the equilibria $(0.35,0.396702)$, where the unstable one is in the interior of the stable one.

From the above results, we can see that for some initial values, both species coexist for model (2.1) in the form of a positive equilibrium $\left(x_{3}, y_{3}\right)$, and for other initial values, both species coexist for model (2.1) in the form of an unstable oscillatory solution or a stable oscillatory solution.

\subsection{Bogdanov-Takens bifurcation}

From Theorem 2.7, we know that system (2.1) has a cusp $\left(\frac{1}{2}, \frac{1}{4}-h\right)$ when $a=\frac{1}{4}, 0<h<\frac{1}{4}$ and $\mu^{2}-4 a d^{2}=0$ (i.e., $\mu=d$ ). 


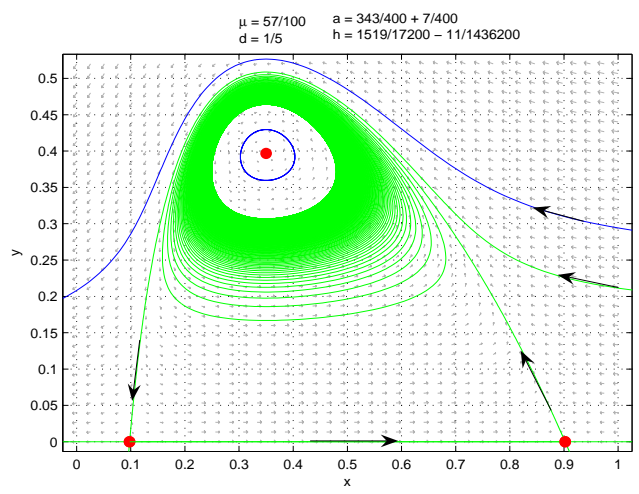

FiguRE 6 . The existence of two limit cycles, where the outer is stable and the inner is unstable.

In this section, we are interested in the bifurcation of the cusp as the parameters vary in a small neighborhood of $\left(a_{0}, d_{0}, \mu_{0}, h_{0}\right)$, where $a_{0}=\frac{1}{4}, \mu_{0}=d_{0}$ and $0<h_{0}<\frac{1}{4}$. We firstly prove this equilibrium is a cusp of codimension 2 (i.e. a Bogdanov-Takens singularity), thus we consider system

$$
\begin{aligned}
& \dot{x}=x(1-x)-\frac{x y}{a_{0}+x^{2}}-h_{0}, \\
& \dot{y}=y\left(\frac{\mu_{0} x}{a_{0}+x^{2}}-d_{0}\right) .
\end{aligned}
$$

First of all, we translate the interior equilibrium $\left(\frac{1}{2}, \frac{1}{4}-h_{0}\right)$ to the origin and expand system (3.9) in a power series around the origin. Let $X=x-\frac{1}{2}, Y=y-\frac{1}{4}+h_{0}$. Then system (3.9) can be rewritten as

$$
\begin{aligned}
& \dot{X}=-Y-\left(\frac{1}{2}+2 h_{0}\right) X^{2}+P_{1}(X, Y), \\
& \dot{Y}=\left(2 \mu_{0} h_{0}-\frac{\mu_{0}}{2}\right) X^{2}+Q_{1}(X, Y),
\end{aligned}
$$

where $P_{1}(X, Y)$ and $Q_{1}(X, Y)$ are $C^{\infty}$ functions at least of order three in $(X, Y)$. Next, we study the normal form of system (3.10) in the two-dimensional center manifold. Introduce a new time variable $\tau$ by $t=-\tau$. Rewriting $\tau$ as $t$, we obtain

$$
\begin{aligned}
& \dot{X}=Y+\left(\frac{1}{2}+2 h_{0}\right) X^{2}+P_{2}(X, Y), \\
& \dot{Y}=-\left(2 \mu_{0} h_{0}-\frac{\mu_{0}}{2}\right) X^{2}+Q_{2}(X, Y) .
\end{aligned}
$$

In order to find the canonical normal form of the cusp, we take

$$
x=X, y=Y+\left(\frac{1}{2}+2 h_{0}\right) X^{2}+P_{2}(X, Y),
$$

then system (3.11) becomes

$$
\begin{aligned}
& \dot{x}=y, \\
& \dot{y}=-\left(2 \mu_{0} h_{0}-\frac{\mu_{0}}{2}\right) x^{2}+\left(1+4 h_{0}\right) x y+R(x, y),
\end{aligned}
$$

where $R(x, y)$ is a $C^{\infty}$ function at least of order three in $(x, y)$. Since

$$
-\left(2 \mu_{0} h_{0}-\frac{\mu_{0}}{2}\right)=2 \mu_{0}\left(\frac{1}{4}-h_{0}\right)>0,1+4 h_{0}>0,
$$

we can see that the interior equilibria $\left(\frac{1}{2}, \frac{1}{4}-h\right)$ of system (3.9) is a cusp of codimension 2 . Then the Theorem 2.7 is completely proved. 
In the following, we discuss if system (2.1) can undergo Bogdanov-Taken bifurcation under a small parameter perturbation if the bifurcation parameters are chosen suitably. Actually, we have the following theorem.

Theorem 3.5. When $a=\frac{1}{4}, \mu=d$, and $0<h<\frac{1}{4}$, system (2.1) has a cusp $F=\left(\frac{1}{2}, \frac{1}{4}-h\right)$ of codimension 2 (i.e., B-T singularity). If we choose a and $d$ as bifurcation parameters, then system (2.1) undergoes $B$-T bifurcation in a small neighborhood of interior equilibrium $F$ as $(a, d)$ varies near $\left(\frac{1}{4}, \mu\right)$. Hence, there exist some parameter values such that system (2.1) has a stable limit cycle, and there exist some other parameter values such that system (2.1) has a stable homoclinic loop.

Proof. We choose $a$ and $d$ as bifurcation parameters. Consider

$$
\begin{aligned}
& \dot{x}=x(1-x)-\frac{x y}{a_{0}+\lambda_{1}+x^{2}}-h_{0}, \\
& \dot{y}=y\left(\frac{\mu_{0} x}{a_{0}+\lambda_{1}+x^{2}}-\left(d_{0}+\lambda_{2}\right)\right),
\end{aligned}
$$

where the constants $a_{0}=\frac{1}{4}, 0<h_{0}<\frac{1}{4}$ and $\mu_{0}=d_{0}$, and $\lambda=\left(\lambda_{1}, \lambda_{2}\right)$ is a parameter vector in a small neighborhood of $(0,0)$. We are only interested in the phase portraits of system (3.13) when $x$ and $y$ are in a small neighborhood of the interior equilibrium $\left(\frac{1}{2}, \frac{1}{4}-h_{0}\right)$. We translate $\left(\frac{1}{2}, \frac{1}{4}-h_{0}\right)$ to the origin and expand system in a power series around the origin. Let

$$
X=x-\frac{1}{2}, Y=y-\frac{1}{4}+h_{0}
$$

then we have

$$
\begin{aligned}
& \dot{X}=a_{1}+a_{2} X+a_{3} Y+a_{4} X^{2}+a_{5} X Y+a_{6} X^{3}+a_{7} X^{2} Y+P_{1}\left(X, Y, \lambda_{1}\right), \\
& \dot{Y}=b_{1}+b_{2} X+b_{3} Y+b_{4} X^{2}+b_{5} X Y+Q_{1}\left(X, Y, \lambda_{1}\right)
\end{aligned}
$$

where $P_{1}\left(X, Y, \lambda_{1}\right)$ and $Q_{1}\left(X, Y, \lambda_{1}\right)$ are $C^{\infty}$ functions at least of the fourth order and third order with respect to $(X, Y)$, respectively, and

$$
\begin{gathered}
a_{1}=\frac{\lambda_{1}\left(1-4 h_{0}\right)}{2\left(1+2 \lambda_{1}\right)}, a_{2}=\frac{\lambda_{1}\left(4 h_{0}-1\right)}{\left(2 \lambda_{1}+1\right)^{2}}, a_{3}=\frac{-1}{2 \lambda_{1}+1}, a_{4}=\frac{\left(1-4 h_{0}\right)\left(1+6 \lambda_{1}\right)}{2\left(2 \lambda_{1}+1\right)^{3}}-1, a_{5}=\frac{-4 \lambda_{1}}{\left(2 \lambda_{1}+1\right)^{2}}, \\
a_{6}=\frac{\left(1-4 h_{0}\right)\left(4 \lambda_{1}^{2}-4 \lambda_{1}-1\right)}{\left(2 \lambda_{1}+1\right)^{2}}, a_{7}=\frac{2\left(6 \lambda_{1}+1\right)}{\left(2 \lambda_{1}+1\right)^{3}}, b_{1}=\frac{\left(4 h_{0}-1\right)\left(2 \lambda_{1} \lambda_{2}+2 d_{0} \lambda_{1}+\lambda_{2}\right)}{4\left(2 \lambda_{1}+1\right)}, \\
b_{2}=\frac{d_{0}\left(1-4 h_{0}\right) \lambda_{1}}{\left(2 \lambda_{1}+1\right)^{2}}, b_{3}=-\frac{2 d_{0} \lambda_{1}}{2 \lambda_{1}+1}-\lambda_{2}, b_{4}=\frac{d_{0}\left(4 h_{0}-1\right)\left(6 \lambda_{1}+1\right)}{2\left(2 \lambda_{1}+1\right)^{3}}, b_{5}=\frac{4 d_{0} \lambda_{1}}{\left(2 \lambda_{1}+1\right)^{2}} .
\end{gathered}
$$

Now, we rewrite (3.14) by setting

$$
\begin{aligned}
& x=X, \\
& y=a_{1}+a_{2} X+a_{3} Y+a_{4} X^{2}+a_{5} X Y+a_{6} X^{3}+a_{7} X^{2} Y+P_{1}\left(X, Y, \lambda_{1}\right),
\end{aligned}
$$

to obtain

$$
\begin{aligned}
& \dot{x}=y, \\
& \dot{y}=c_{1}+c_{2} x+c_{3} y+c_{4} x^{2}+c_{5} x y+c_{6} y^{2}+R_{1}\left(x, y, \lambda_{1}, \lambda_{2}\right),
\end{aligned}
$$

where $R_{1}\left(x, y, \lambda_{1}, \lambda_{2}\right)$ is a $C^{\infty}$ function at least of the third order with respect to $(x, y)$, and

$$
\begin{gathered}
c_{1}=a_{3} b_{1}-a_{1} b_{3}, c_{2}=-\frac{-a_{1}^{2} a_{5}^{2}+2 a_{1}^{2} a_{3} a_{7}-a_{3}^{2} a_{5} b_{1}-a_{3}^{3} b_{2}+a_{2} a_{3}^{2} b_{3}+a_{1} a_{3}^{2} b_{5}}{a_{3}^{2}}, \\
c_{3}=-\frac{-a_{2} a_{3}+a_{1} a_{5}-a_{3} b_{3}}{a_{3}},
\end{gathered}
$$




$$
\begin{gathered}
c_{4}=-\frac{a_{1} a_{3} a_{4} a_{5}-a_{1} a_{2} a_{5}^{2}-3 a_{1} a_{3}^{2} a_{6}+2 a_{1} a_{2} a_{3} a_{7}-a_{3}^{2} a_{7} b_{1}-a_{3}^{2} a_{5} b_{2}+a_{3}^{2} a_{4} b_{3}-a_{3}^{3} b_{4}+a_{2} a_{3}^{2} b_{5}}{a_{3}^{2}}, \\
c_{5}=-\frac{-2 a_{3}^{2} a_{4}+a_{2} a_{3} a_{5}+a_{1} a_{5}^{2}-2 a_{1} a_{3} a_{7}-a_{3}^{2} b_{5}}{a_{3}^{2}}, c_{6}=\frac{a_{5}}{a_{3}} .
\end{gathered}
$$

Next, introducing a new time variable $\tau$ by $d t=\left(1-c_{6} x\right) d \tau$, rewriting $\tau$ as $t$, we obtain

$$
\begin{aligned}
& \dot{x}=y\left(1-c_{6} x\right), \\
& \dot{y}=\left(1-c_{6} x\right)\left(c_{1}+c_{2} x+c_{3} y+c_{4} x^{2}+c_{5} x y+c_{6} y^{2}+R_{1}\left(x, y, \lambda_{1}, \lambda_{2}\right)\right) .
\end{aligned}
$$

Then let $X=x, Y=y\left(1-c_{6} x\right)$, we have

$$
\begin{aligned}
& \dot{X}=Y \\
& \dot{Y}=d_{1}+d_{2} X+d_{3} Y+d_{4} X^{2}+d_{5} X Y+R_{2}\left(X, Y, \lambda_{1}, \lambda_{2}\right)
\end{aligned}
$$

where $R_{2}\left(X, Y, \lambda_{1}, \lambda_{2}\right)$ is a $C^{\infty}$ function at least of the third order with respect to $(X, Y)$, and

$$
d_{1}=c_{1}, d_{2}=c_{2}-2 c_{1} c_{6}, d_{3}=c_{3}, d_{4}=c_{4}-2 c_{2} c_{6}+c_{1} c_{6}^{2}, d_{5}=c_{5}-c_{3} c_{6} .
$$

We can check that $d_{4}>0$ and $d_{5}<0$ when $\lambda_{i}$ are sufficiently small. Making the following change of variables

$$
x=X, y=\frac{Y}{\sqrt{d_{4}}}, \tau=\sqrt{d_{4}} t
$$

and still denoting $\tau$ by $t$, then we obtain

$$
\begin{aligned}
& \dot{x}=y, \\
& \dot{y}=e_{1}+e_{2} x+e_{3} y+x^{2}+e_{4} x y+R_{3}\left(x, y, \lambda_{1}, \lambda_{2}\right),
\end{aligned}
$$

where

$$
e_{1}=\frac{d_{1}}{d_{4}}, e_{2}=\frac{d_{2}}{d_{4}}, e_{3}=\frac{d_{3}}{\sqrt{d_{4}}}, e_{4}=\frac{d_{5}}{\sqrt{d_{4}}} .
$$

Make the change of variables $X=x+\frac{e_{2}}{2}, Y=y$, we rewrite (3.18) as

$$
\begin{aligned}
& \dot{X}=Y, \\
& \dot{Y}=\gamma_{1}+\gamma_{2} Y+X^{2}+\gamma_{3} X Y+R_{4}\left(X, Y, \lambda_{1}, \lambda_{2}\right),
\end{aligned}
$$

where $R_{4}\left(X, Y, \lambda_{1}, \lambda_{2}\right)$ is a $C^{\infty}$ function at least of the third order with respect to $(X, Y)$, and

$$
\gamma_{1}=e_{1}-\frac{e_{2}^{2}}{4}, \gamma_{2}=e_{3}-\frac{e_{2} e_{4}}{2}, \gamma_{3}=e_{4}
$$

Since $\gamma_{3}<0$ when $\lambda_{i}$ are small. Make the change of variables one more time by setting

$$
x=\gamma_{3}^{2} X, y=-\gamma_{3}^{3} Y, \tau=-\frac{t}{\gamma_{3}},
$$

and still denoting $\tau$ by $t$, we finally have

$$
\begin{aligned}
& \dot{x}=y, \\
& \dot{y}=\xi_{1}+\xi_{2} y+x^{2}-x y+R_{5}\left(x, y, \lambda_{1}, \lambda_{2}\right),
\end{aligned}
$$

where $R_{5}\left(x, y, \lambda_{1}, \lambda_{2}\right)$ is a $C^{\infty}$ function at least of the third order with respect to $(x, y)$, and

$$
\xi_{1}=\gamma_{1} \gamma_{3}^{4}, \xi_{2}=-\gamma_{2} \gamma_{3}
$$


We can express $\xi_{1}$ and $\xi_{2}$ in terms of $\lambda_{1}$ and $\lambda_{2}$ as follows

$$
\begin{aligned}
& \xi_{1}=s_{1} \lambda_{1}+s_{2} \lambda_{2}+s_{3} \lambda_{1}^{2}+s_{4} \lambda_{1} \lambda_{2}+s_{5} \lambda_{2}^{2}+o\left(\left|\lambda_{1}, \lambda_{2}\right|^{2}\right) \\
& \xi_{2}=t_{1} \lambda_{1}+t_{2} \lambda_{2}+t_{3} \lambda_{1}^{2}+t_{4} \lambda_{1} \lambda_{2}+t_{5} \lambda_{2}^{2}+o\left(\left|\lambda_{1}, \lambda_{2}\right|^{2}\right)
\end{aligned}
$$

where

$$
\begin{gathered}
s_{1}=\frac{4\left(1+4 h_{0}\right)^{4}}{d_{0}^{2}\left(4 h_{0}-1\right)^{2}}, s_{2}=\frac{2\left(1+4 h_{0}\right)^{4}}{d_{0}^{3}\left(4 h_{0}-1\right)^{2}}, s_{3}=-\frac{4\left(4 h_{0}+1\right)^{3}\left(16 d_{0}^{2}\left(4 h_{0}-1\right)+9\left(4 h_{0}-1\right)^{2}\left(4 h_{0}+1\right)+d\left(23-16 h_{0}+80 h_{0}^{2}\right)\right)}{d_{0}^{3}\left(4 h_{0}-1\right)^{3}}, \\
s_{4}=\frac{2\left(4 h_{0}+1\right)^{3}\left(16 d_{0}^{2}\left(4 h_{0}-1\right)+9\left(4 h_{0}-1\right)^{2}\left(4 h_{0}+1\right)+d\left(38+32 h_{0}+32 h_{0}^{2}\right)\right)}{d_{0}^{4}\left(4 h_{0}-1\right)^{3}}, s_{5}=-\frac{12\left(4 h_{0}+1\right)^{4}}{d_{0}^{4}\left(4 h_{0}-1\right)^{3}}, t_{1}=\frac{4\left(d_{0}+1\right)\left(4 h_{0}+1\right)}{d\left(4 h_{0}-1\right)}, \\
t_{2}=\frac{2\left(4 h_{0}+1\right)}{d\left(4 h_{0}-1\right)}, t_{3}=-\frac{8\left(8 d_{0}^{2} h_{0}\left(4 h_{0}+1\right)+2 d_{0}^{3}\left(4 h_{0}-1\right)+2\left(4 h_{0}-1\right)^{2}\left(4 h_{0}^{2}+5 h_{0}+1\right)+d_{0}\left(64 h_{0}^{3}-16 h_{0}^{2}+4 h_{0}+7\right)\right)}{d_{0}^{2}\left(4 h_{0}-1\right)^{2}}, \\
t_{4}=-\frac{2\left(11+28 h_{0}-48 h_{0}^{2}+64 h_{0}^{3}+4 d_{0}^{2}\left(4 h_{0}-1\right)+4 d_{0}\left(4 h_{0}+3\right)\right)}{d_{0}^{2}\left(4 h_{0}-1\right)^{2}}, t_{5}=-\frac{4\left(4 h_{0}+1\right)}{d_{0}^{2}\left(4 h_{0}-1\right)^{2}} .
\end{gathered}
$$

Note that

$$
\left|\frac{\partial\left(\xi_{1}, \xi_{2}\right)}{\partial\left(\lambda_{1}, \lambda_{2}\right)}\right|_{\lambda=0}=-\frac{8\left(1+4 h_{0}\right)^{5}}{d_{0}^{4}\left(4 h_{0}-1\right)^{3}}>0
$$

since $0<h_{0}<\frac{1}{4}$. Thus the parameter transformation (3.21) is a homeomorphism in a small neighborhood of the origin, and $\xi_{1}$ and $\xi_{2}$ are independent parameters.

By the results in Bogdanov [2,3] and Takens [15] or Perko [13], we know that system (3.20) undergoes the Bogdanov-Takens bifurcation when $\lambda$ is in a small neighborhood of the origin. The local representations of the bifurcation curves up to second-order approximations are as follows:

(1) The saddle-node bifurcation curve $S N=\left\{\left(\xi_{1}, \xi_{2}\right): \xi_{1}=0, \xi_{2} \neq 0\right\}$, i.e.,

$$
\begin{aligned}
S N & =\left\{\left(\lambda_{1}, \lambda_{2}\right): \frac{4\left(1+4 h_{0}\right)^{4}}{d_{0}^{2}\left(4 h_{0}-1\right)^{2}} \lambda_{1}+\frac{2\left(1+4 h_{0}\right)^{4}}{d_{0}^{3}\left(4 h_{0}-1\right)^{2}} \lambda_{2}-\frac{12\left(4 h_{0}+1\right)^{4}}{d_{0}^{4}\left(4 h_{0}-1\right)^{3}} \lambda_{2}^{2}\right. \\
& +\frac{2\left(4 h_{0}+1\right)^{3}\left(16 d_{0}^{2}\left(4 h_{0}-1\right)+9\left(4 h_{0}-1\right)^{2}\left(4 h_{0}+1\right)+d\left(38+32 h_{0}+32 h_{0}^{2}\right)\right)}{d_{0}^{4}\left(4 h_{0}-1\right)^{3}} \lambda_{1} \lambda_{2} \\
& \left.-\frac{4\left(4 h_{0}+1\right)^{3}\left(16 d_{0}^{2}\left(4 h_{0}-1\right)+9\left(4 h_{0}-1\right)^{2}\left(4 h_{0}+1\right)+d\left(23-16 h_{0}+80 h_{0}^{2}\right)\right)}{d_{0}^{3}\left(4 h_{0}-1\right)^{3}} \lambda_{1}^{2}=0, \xi_{2} \neq 0\right\} .
\end{aligned}
$$

(2) The Hopf bifurcation curve $H=\left\{\left(\xi_{1}, \xi_{2}\right): \xi_{2}=-\sqrt{-\xi_{1}}, \xi_{1}<0\right\}$, i.e.,

$$
\begin{aligned}
H= & \left\{\left(\lambda_{1}, \lambda_{2}\right): \frac{4\left(4 h_{0}+1\right)^{4}}{d_{0}^{2}\left(4 h_{0}-1\right)^{2}} \lambda_{1}+\frac{2\left(4 h_{0}+1\right)^{4}}{d_{0}^{3}\left(4 h_{0}-1\right)^{2}} \lambda_{2}-\frac{4\left(4 h_{0}+1\right)^{2}\left(d_{0}^{2}\left(1-4 h_{0}\right)+3\left(4 h_{0}+1\right)^{2}\right)}{d_{0}^{4}\left(4 h_{0}-1\right)^{3}} \lambda_{2}^{2}\right. \\
& +\frac{2\left(4 h_{0}+1\right)^{2}}{d_{0}^{4}\left(4 h_{0}-1\right)^{3}}\left(8 d_{0}^{3}\left(4 h_{0}-1\right)+9\left(4 h_{0}-1\right)^{2}\left(4 h_{0}+1\right)^{2}+8 d_{0}^{2}\left(32 h_{0}^{2}+4 h_{0}-3\right)+\right. \\
& \left.2 d_{0}\left(64 h_{0}^{3}+80 h_{0}^{2}+92 h_{0}+19\right)\right) \lambda_{1} \lambda_{2}-\frac{4\left(4 h_{0}+1\right)^{2}}{d_{0}^{3}\left(4 h_{0}-1\right)^{3}}\left(4 d_{0}^{3}\left(1-4 h_{0}\right)+9\left(4 h_{0}-1\right)^{2}\left(4 h_{0}+1\right)^{2}+\right. \\
& \left.\left.8 d_{0}^{2}\left(32 h_{0}^{2}-4 h_{0}-1\right)+d_{0}\left(320 h_{0}^{3}+16 h_{0}^{2}+60 h_{0}+27\right)\right) \lambda_{1}^{2}=0, \xi_{1}<0, \xi_{2}<0\right\} .
\end{aligned}
$$

(3) The homoclinic bifurcation curve $H L=\left\{\left(\xi_{1}, \xi_{2}\right): \xi_{2}=-\frac{5}{7} \sqrt{-\xi_{1}}, \xi_{1}<0\right\}$, i.e.,

$$
\begin{aligned}
H L= & \left\{\left(\lambda_{1}, \lambda_{2}\right): \frac{100\left(4 h_{0}+1\right)^{4}}{49 d_{0}^{2}\left(4 h_{0}-1\right)^{2}} \lambda_{1}+\frac{50\left(4 h_{0}+1\right)^{4}}{49 d_{0}^{3}\left(4 h_{0}-1\right)^{2}} \lambda_{2}+\frac{196 d_{0}^{2}\left(4 h_{0}+1\right)^{2}\left(4 h_{0}-1\right)-300\left(4 h_{0}+1\right)^{4}}{49 d_{0}^{4}\left(4 h_{0}-1\right)^{3}} \lambda_{2}^{2}\right. \\
& +\frac{2\left(4 h_{0}+1\right)^{2}}{49 d_{0}^{4}\left(4 h_{0}-1\right)^{3}}\left(392 d_{0}^{3}\left(4 h_{0}-1\right)+225\left(4 h_{0}-1\right)^{2}\left(4 h_{0}+1\right)^{2}+8 d_{0}^{2}\left(800 h_{0}^{2}+196 h_{0}-99\right)\right. \\
& \left.+50 d_{0}\left(64 h_{0}^{3}+80 h_{0}^{2}+92 h_{0}+19\right)\right) \lambda_{1} \lambda_{2}-\frac{4\left(4 h_{0}+1\right)^{2}}{49 d_{0}^{3}\left(4 h_{0}-1\right)^{3}}\left(-196 d_{0}^{3}\left(4 h_{0}-1\right)+225\left(4 h_{0}-1\right)^{2}\left(4 h_{0}+1\right)^{2}\right. \\
& \left.\left.+8 d_{0}^{2}\left(800 h_{0}^{2}-196 h_{0}-1\right)+d_{0}\left(8000 h_{0}^{3}+400 h_{0}^{2}+1116 h_{0}+771\right)\right) \lambda_{1}^{2}=0, \xi_{1}<0, \xi_{2}<0\right\} .
\end{aligned}
$$

The bifurcation diagram and phase portraits of system (3.13) with $h_{0}=\frac{1}{8}, \mu_{0}=d_{0}=\frac{1}{2}$ and $a_{0}=\frac{1}{4}$ are given in Fig. 7 and Fig. 8, respectively. These bifurcation curves $H, H L$, and $S N$ divide the small neighborhood of the origin in the parameter plane $\left(\lambda_{1}, \lambda_{2}\right)$ into four regions (see Fig. 7 ). 
(a) When $\left(\lambda_{1}, \lambda_{2}\right)=(0,0)$, the unique positive equilibrium is a cusp of codimension 2 (see Fig. 8(a)).

(b) There are no positive equilibria when the parameters lie in region I (see Fig. 8(b)), we can see that there exists one separatrix which converges to the boundary equilibrium $A$. The prey species will tend to extinction as the initial value density lies in the left of the separatrix, which in turn lead to the extinction of the predator. The predator species will tend to extinction as the initial value density lies in the right of the separatrix.

(c) When the parameters lie on curve $S N$, there is a positive equilibrium, which is a saddle-node.

(d) Two positive equilibria, which are a stable focus and a saddle, will occur through the saddle-node bifurcation when the parameters cross $S N$ into region II (see Fig. 8(c)).

(e) A stable limit cycle will appear through the supercritical Hopf bifurcation when the parameters cross $H$ into region III (see Fig. 8(d)), where the focus is unstable, whereas the focus is a stable multiple one with multiplicity one when the parameters lie on the curve $H$.

(f) A stable homoclinic cycle will occur through the homoclinic bifurcation when the parameters pass region III and lie on curve $H L$ (see Fig. 8(e)).

(g) The relative location of one stable and one unstable manifold of the saddle $D\left(x_{2}, y_{2}\right)$ will be switched when the parameters cross III into region IV (compare Fig. 8(d) and Fig. 8(f)).

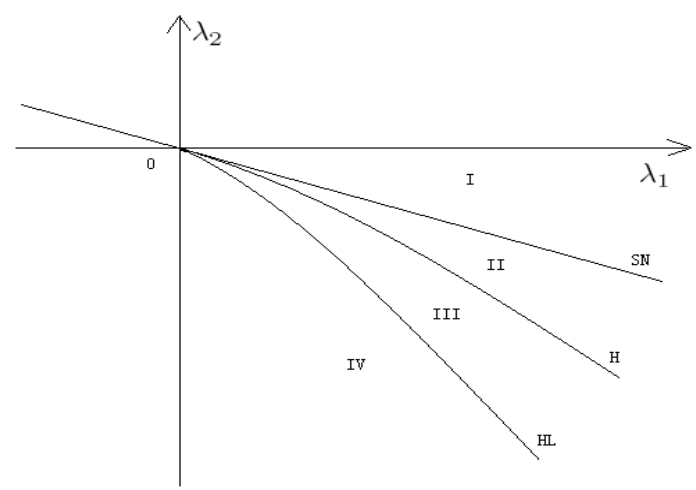

Figure 7 . The bifurcation diagram of system (3.13) with $h_{0}=\frac{1}{8}, \mu_{0}=d_{0}=\frac{1}{2}$ and $a_{0}=\frac{1}{4}$.

When the predator death rate $d$ tends to the predator conversion rate $\mu$ and the half saturation constant $a$ tends to $\frac{1}{4}$, the above results say that if the constant-yield harvesting rate $h$ satisfies $0<h<\frac{1}{4}$, then there exist some values of parameters such that the prey and predator coexist in the form of a positive equilibrium or a periodic orbit with a finite period for different initial values, respectively. And there exist other values of parameters such that the prey and predator coexist in the form of a positive equilibrium or a periodic orbit with an infinite period for different initial values, respectively.

\section{Periodic solutions and invariant tori with seasonal harvesting}

In this section, in order to investigate the effect of seasonal harvesting on the dynamics of system (2.1), we now study model (1.2) to reflect the fact that harvesting does not always occur at a constant-yield or constant-effort. For example, populations of many species of fish are harvested at a higher rate in warmer seasons than in colder months. So we assume that the interacting species are both harvested at a periodic rate. Thus the prey (or predators) harvesting reaches a maximum rate $h+\gamma_{1}$ (or $2 \gamma_{2}$ ) at time $t=\frac{r}{4}+n$ where $n$ is an integer (representing the year), and a minimum value $h-\gamma_{1}$ (or 0 ) when $t=\frac{3 r}{4}+n$, exactly one-half year later $([9])$. 
(a)

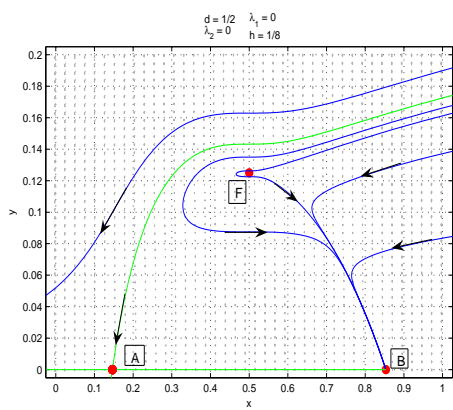

(d)

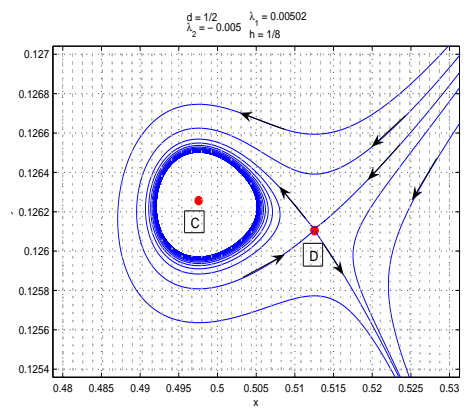

(b)

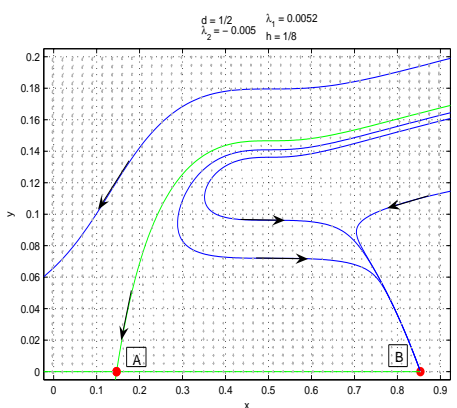

(e)

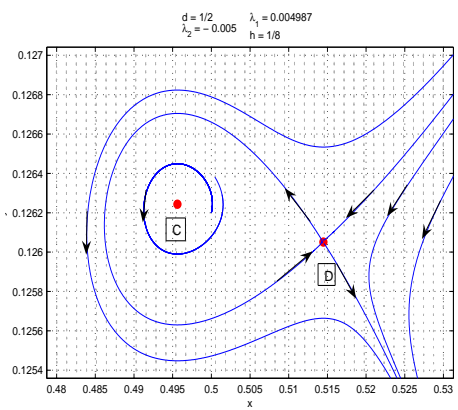

(c)

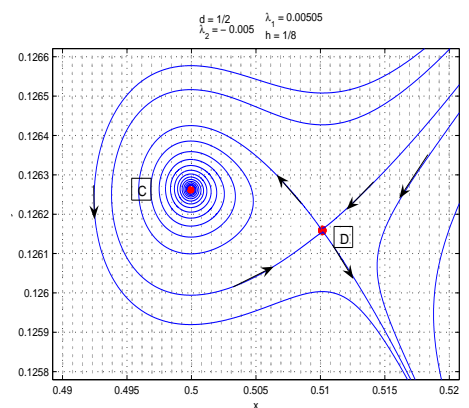

(f)

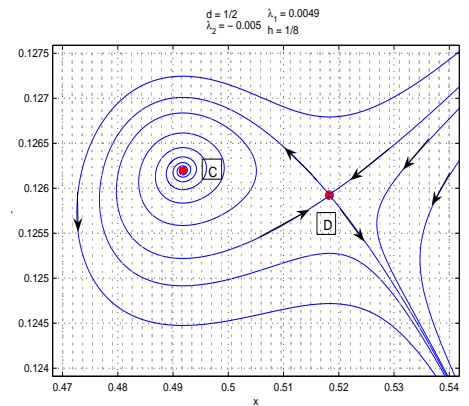

FigurE 8. Phase portraits of system (3.13) with $h_{0}=\frac{1}{8}, \mu_{0}=d_{0}=\frac{1}{2}$ and $a_{0}=\frac{1}{4}$. (a) A cusp of codimension 2 when $\left(\lambda_{1}, \lambda_{2}\right)=(0,0)$; (b) No positive equilibria when $\left(\lambda_{1}, \lambda_{2}\right)=$ $(0.0052,-0.005)$ lies in region I; (c) A stable focus when $\left(\lambda_{1}, \lambda_{2}\right)=(0.00505,-0.005)$ lies in region II; (d) A stable limit cycle when $\left(\lambda_{1}, \lambda_{2}\right)=(0.00502,-0.005)$ lies in region III; (e) A stable homoclinic cycle when $\left(\lambda_{1}, \lambda_{2}\right)=(0.004987,-0.005)$ lies on curve HL; (f) An unstable focus when $\left(\lambda_{1}, \lambda_{2}\right)=(0.0049,-0.005)$ lies in region IV.

In the following, we consider the existence of an asymptotically stable periodic solution and the bifurcation of a stable periodic orbit into an invariant torus by theoretical analysis and numerical simulations in system (1.2), respectively.

Firstly, we rewrite system (1.2) as follows

$$
\dot{Y}=f(Y)+\epsilon g(t, Y)
$$

where $Y=(x, y)^{T}, f(Y)=\left(x(1-x)-\frac{x y}{a+x^{2}}-h, y\left(\frac{\mu x}{a+x^{2}}-d\right)\right)^{T}, g(t, Y)=\left(-\sin \left(\frac{2 \pi}{r} t\right),-\left(1+\sin \left(\frac{2 \pi}{r} t\right)\right)\right)^{T}$ and $\epsilon=\left(\gamma_{1}, \gamma_{2}\right)$.

\subsection{Existence of asymptotically stable periodic solutions}

The following lemma is Theorem 2 in [4].

Lemma 4.1. Let $f(Y)$ and $g(t, Y)$ be continuously differentiable with respect to the components of $Y$ and let $g(t, Y)$ be periodic in $t$ with period $w$ for each $Y$. Let $Y_{\infty}$ be a critical point of system (4.1) when $\epsilon=0$, i.e. a solution of the equation $f(Y)=0$, which is asymptotically stable in the strong sense that the eigenvalues of the matrix $f_{Y}\left[Y_{\infty}\right]$ all have negative real parts. Then the perturbed system (4.1) has 
an asymptotically stable periodic solution $p(t, \epsilon)$ of the same period $w$ for all sufficiently small $\epsilon$, with $\lim _{\epsilon \longrightarrow 0} p(t, \epsilon)=Y_{\infty}$

Applying Theorem 2.8 with the Lemma 4.1, we have the following theorem about the existence of an asymptotically stable periodic solution in system (1.2).

Theorem 4.2. If $\mu^{2}-4 a d^{2}>0$ and $0<h<\min \left\{h_{1}, h_{3}\right\}$, then the system (1.2) has an asymptotically stable periodic solution $p\left(t, \gamma_{1}, \gamma_{2}\right)$ of period $r$ for all sufficiently small $\left(\gamma_{1}, \gamma_{2}\right)$, with $\lim _{\left(\gamma_{1}, \gamma_{2}\right) \longrightarrow 0} p\left(t, \gamma_{1}, \gamma_{2}\right)=$ $\left(x_{3}, y_{3}\right)$.

In Fig. 6(b), there exists a hyperbolic stable focus. Now we plot the phase portrait and the corresponding attractor of the Poincaré map of system (1.2) with $r=1$ in $(x, y)$-plane in Fig. 9(a) and Fig. 9(b), respectively, where $\gamma_{1}=\gamma_{2}=0.006$ and the other parameter values are the same as those in Fig. 6(b), that is $a=0.5, h=0.015, \mu=1.35$ and $d=0.5$. We choose the initial density as $\left(x_{0}, y_{0}\right)=(0.2,0.4)$, which is located in the attraction basin of the stable focus of Fig. 6(b). The attractor of the Poincaré map is a fixed point (see Fig. 9(b)), which corresponds to the asymptotically stable periodic orbit of the system (1.2) (see Fig. 9(a)). The time series of the predator corresponding to Fig. 9(a) is given in Fig. $9(\mathrm{c})$.

(a)

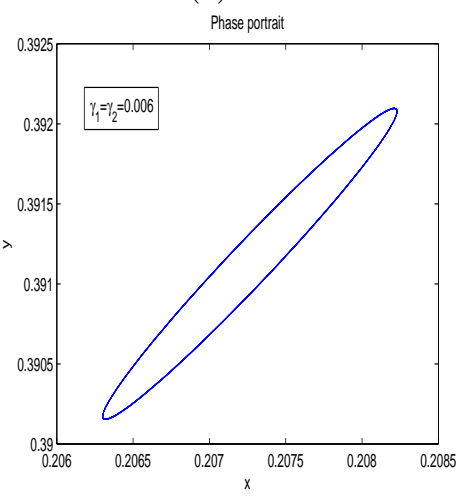

(b)

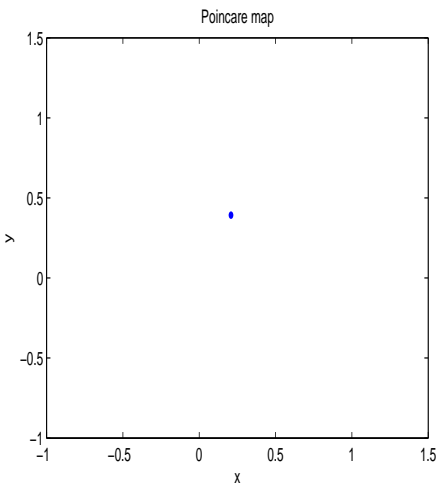

(c)

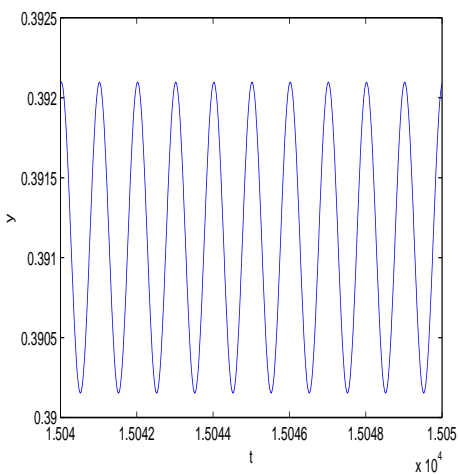

Figure 9. (a) The phase portrait of system (1.2) with $r=1, a=0.5, h=0.015, \mu=$ $1.35, d=0.5, \gamma_{1}=\gamma_{2}=0.006$ and initial value $\left(x_{0}, y_{0}\right)=(0.2,0.4)$; (b) An attractor of the Poincaré map corresponding to (a); (c) The time series of the predator corresponding to $(\mathrm{a})$.

\subsection{Bifurcation of stable periodic solutions into invariant tori}

From Theorem 3.2, we know that the interior equilibrium $\left(x_{3}, y_{3}\right)$ of system $(2.1)$ is a stable weak focus of multiplicity 1 when $\mu^{2}-4 a d^{2}>0,0<h=h_{3}<h_{1}$ and $L_{11}<0$.

Applying Theorems 2.8, 3.2 in previous sections with the Theorem 6.3 in section 12 in Chow and Hale [7], we have the following theorem about the existence of an asymptotically stable invariant torus in system (1.2).

Theorem 4.3. If $\mu^{2}-4 a d^{2}>0,0<h-h_{3} \ll 1$ and $L_{11}<0$, then system (2.1) has a stable limit cycle enclosing $\left(x_{3}, y_{3}\right)$ by the supercritical Hopf bifurcation. If the following nonresonant conditions

$$
r \neq 2 k \pi, \quad k \in N^{+},
$$




$$
m+n \frac{2 \pi}{r} \neq 0, \quad 0<|m|+|n| \leq 4, \quad m, n \in N
$$

are also satisfied, then system (1.2) has an asymptotically stable invariant torus.

Proof. We need to check the following three conditions in Theorem 6.3 in section 12 of Chow and Hale [7]:

$$
\begin{gathered}
\beta_{0} \neq 0, \\
\operatorname{det}\left(e^{B T}-I\right) \neq 0, \\
m+n \frac{2 \pi}{T} \neq 0, \quad 0<|m|+|n| \leq 4, \quad m, n \in N,
\end{gathered}
$$

where $\beta_{0}$ is the first Lyapunov constant for the center-type equilibrium $\left(x_{3}, y_{3}\right)$, and it is equivalent to $L_{11}$ in system (2.1); $T$ is the period of periodic perturbation, and $T=r$ in system $(1.2) ; B=\left(\begin{array}{cc}0 & 1 \\ -1 & 0\end{array}\right)$, $I$ is identical matrix. It is easy to check the above conditions for system (1.2).

In Fig. 6(a), there exist an unstable focus and a stable limit cycle enclosing it. Now we plot the bifurcation diagram of system (1.2) with $\gamma_{1}=\gamma_{2}$ and $r=1$ in $\left(\gamma_{1}, x\right)$-plane in Fig. 10, where the other parameter values are the same as those in Fig. 6(a), that is $a=0.4, h=0.065, \mu=0.7$ and $d=0.5$. We choose the initial density as $\left(x_{0}, y_{0}\right)=(0.4,0.25)$, which is located in the attraction basin of the stable limit cycle of Fig. 6(a). The phase portrait, the attractor of the Poincaré map and the time series for $\gamma_{1}=\gamma_{2}=0.0035$ in Fig. 10 are shown in Fig. 11(a), (b) and (c), respectively, from which we can see that the stable limit cycle in Fig. 6(a) is transformed into an attracting invariant torus in Fig. 10. Actually, we can see that the species are always attracted into the invariant torus whenever the initial densities are located in the attraction basin of the stable limit cycle in Fig. 6(a) and the amplitude of the periodic harvesting $\gamma_{1}=\gamma_{2}$ is smaller than 0.004. The invariant torus changes to a periodic orbit when $\gamma$ is larger than 0.004 (see Fig. 12(a), (b) and (c)).

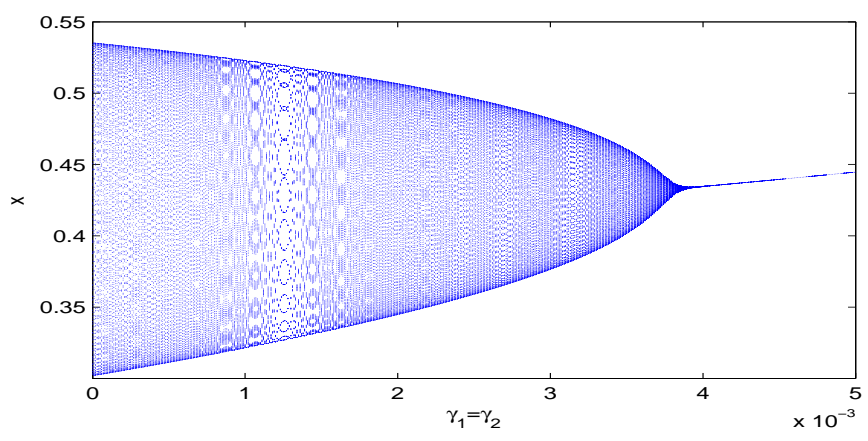

Figure 10. Bifurcation diagram of system (1.2) in terms of $\gamma_{1}=\gamma_{2}$ with $r=1, a=$ $0.4, h=0.065, \mu=0.7, d=0.5$ and initial value $\left(x_{0}, y_{0}\right)=(0.4,0.25)$.

\section{Conclusions}

We have shown that various bifurcations occur in model (1.2) with only constant-yield prey harvesting, including saddle-node bifurcation, degenerate Hopf bifurcation, and Bogdanov-Takens bifurcation (i.e., cusp bifurcation of codimension 2), as the model parameters vary. We then considered system (1.2) with seasonal harvesting in both species and gave sufficient conditions for the existence of an asymptotically stable periodic solution and bifurcation of a stable periodic orbit into a stable invariant torus of the model. 
(a)

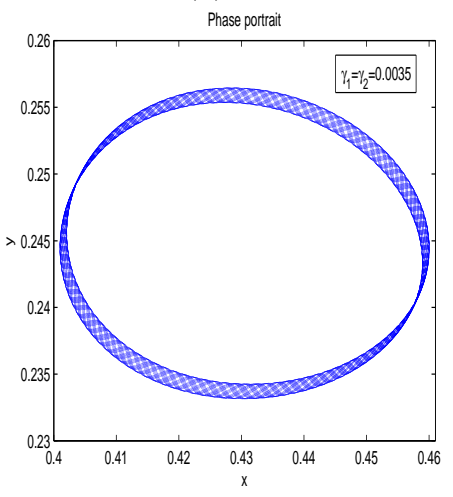

(b)

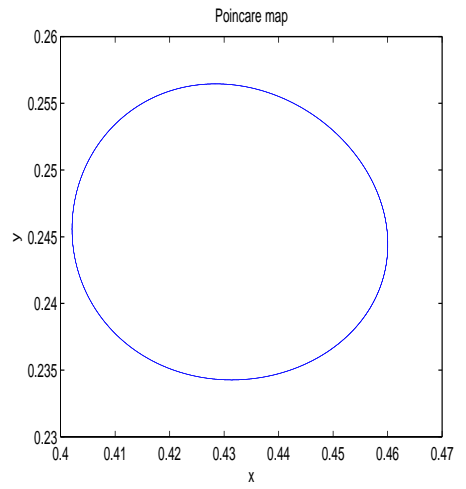

(c)

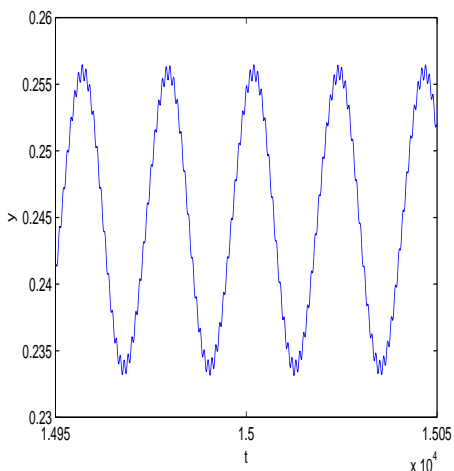

Figure 11. (a) Phase portrait for $\gamma_{1}=\gamma_{2}=0.0035$ in Fig. 4.2 ; (b) An attractor of the Poincaré map corresponding to (a); (c) The time series of the predator corresponding to (a).

(a)

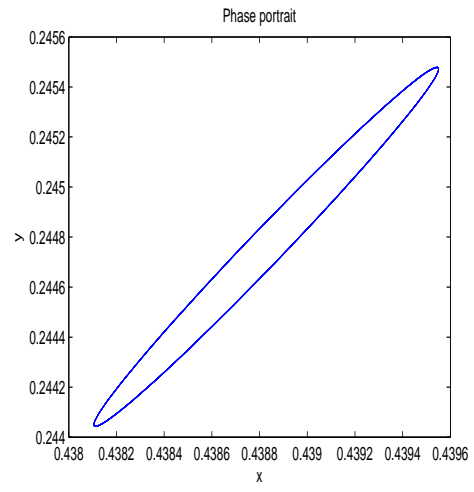

(b)

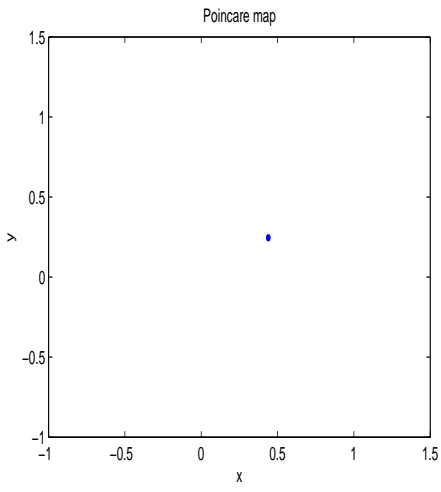

(c)

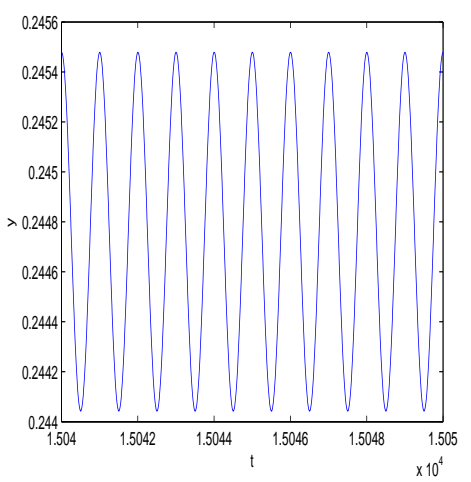

Figure 12. (a) Phase portrait for $\gamma_{1}=\gamma_{2}=0.0045$ in Fig. 4.2; (b) An attractor of the Poincaré map corresponding to (a); (c) The time series of the predator corresponding to (a).

Numerical simulations of the model (1.2) with seasonal harvesting, including bifurcation diagrams, phase portraits and Poincaré maps, were carried out. It was shown that the model undergoes bifurcations from a hyperbolic stable equilibrium to a stable periodic solution and from a stable limit cycle to an invariant torus, respectively, as the amplitude of seasonal harvesting increases.

The results in this paper indicate that the initial species densities are very important for the coexistence of the interacting species when they are both subjected to periodic harvesting. To have the survival of the interacting species with seasonal harvesting in the form of a stable periodic solution or stable quasiperiodic solutions, the initial species densities which are located in the attraction basin of the stable attractor (hyperbolic stable equilibrium or stable limit cycle) in the model with no harvesting or with constant-yield harvesting should be chosen properly.

Acknowledgements. We would like to thank Professor Shigui Ruan for his helpful comments and valuable suggestions. We also thank the referee whose suggestions led to improvements in the manuscript.

Research was partially supported by the National Natural Science Foundation of China (No.11101170, No.11228104), the Research Project of the Central China Normal University (No.CCNU12A01007), and the 
State Scholarship Fund of the China Scholarship Council (No.2011842509).

Research was partially supported by the National Natural Science Foundation of China (No. 11001041) and the State Scholarship Fund of the China Scholarship Council (No.2011662521).

\section{References}

[1] J. F. Andrews. A mathematical model for the continuous culture of microorganisms utilizing inhibitory substrates. Biotechnol. Bioeng., 10 (1968), 707-723.

[2] R. Bogdanov. Bifurcations of a limit cycle for a family of vector fields on the plane. Selecta Math. Soviet. 1 (1981), 373-388.

[3] R. Bogdanov. Versal deformations of a singular point on the plane in the case of zero eigen-values. Selecta Math. Soviet. 1 (1981), 389-421.

[4] F. Brauer. Periodic solutions of some ecological models. J. Theor. Biol. 69 (1977), 143-152.

[5] F. Brauer, D. A Sánchez. Periodic environments and periodic harvesting. Natural Resource Modeling. 16(3) (2003), 233-244.

[6] J. B. Collings. The effects of the functional response on the bifurcation behavior of a mite predator-prey interaction model. J. Math. Biol., 36 (1997), 149-168.

[7] S.-N. Chow, J. K. Hale. Methods of Bifurcation Theory. Springer-Verlag, Berlin-Heidelberg-New York, 1982.

[8] R. M. Etoua, C. Rousseau. Bifurcation analysis of a Generalissed Gause model with prey harvesting and a generalized Holling response function of type III. J. Differential Equations, 249 (2010), 2316-2356.

[9] M. W. Hirsch, S. Smale, R. L. Devaney. Differential Equations, Dynamical Systems and An Introduction to Chaos. Elsevier, California, 2004.

[10] E. Hammill, O. L. Petchey, B. R. Anholt. Predator functional response changed by induced defenses in prey. The American Naturalist, 176(6) (2010), 723-731.

[11] Y. Lamontagne, C. Coutu, C. Rousseau. Bifurcation analysis of a predator-prey system with generalized Holling type III functional response. J. Dynam. Differential Equations. 20 (2008), 535-571.

[12] R. May, J. R. Beddington, C. W. Clark, S. J. Holt, R. M. Laws. Management of multispecies fisheries. Science, 205 (1979), 267-277.

[13] L. Perko. Differential Equations and Dynamical Systems. Springer, New York, 1996.

[14] S. Ruan, D. Xiao. Global analysis in a predator-prey system with nonmonotonic functional response. SIAM J. Appl. Math., 61(4) (2001), 1445-1472.

[15] F. Takens. Forced oscillations and bifurcation, in "Applications of Global Analysis I". Comm. Math. Inst. Rijksuniversitat Utrecht. 3 (1974), 1-59.

[16] R. J. Taylor. Predation. Chapman and Hall, New York, 1984.

[17] G. S. K. Wolkowicz. Bifurcation analysis of a predator-prey system involving group defence. SIAM J. Appl. Math. 48 (1988), 592-606.

[18] D.Xiao, H.Zhu. Multiple focus and hopf bifurcations in a predaotr-prey system with nonmonotonic functional response. SIAM J. Appl. Math. 66 (2006), 802-819.

[19] H. Zhu, S. A. Campbell, G. S. K. Wolkowicz. Bifurcation analysis of a predator-prey system with nonmonotonic functional response. SIAM J. Appl. Math. 63 (2002), 636-682.

[20] Z. Zhang, T. Ding, W. Huang, Z. Dong. Qualitative Theory of Differential Equation. Transl. Math. Monogr. Vol. 101, Amer. Math. Soc. Providence, RI, 1992. 